\title{
Riemannian metrics for neural networks I: Feedforward networks
}

\author{
Yann Ollivier
}

February 4, 2015

\begin{abstract}
We describe four algorithms for neural network training, each adapted to different scalability constraints. These algorithms are mathematically principled and invariant under a number of transformations in data and network representation, from which performance is thus independent. These algorithms are obtained from the setting of differential geometry, and are based on either the natural gradient using the Fisher information matrix, or on Hessian methods, scaled down in a specific way to allow for scalability while keeping some of their key mathematical properties.
\end{abstract}

The most standard way to train neural networks, backpropagation, has several known shortcomings. Convergence can be quite slow. Backpropagation is sensitive to data representation: for instance, even such a simple operation as exchanging 0 's and 1's on the input layer will affect performance (Figure 11), because this amounts to changing the parameters (weights and biases) in a non-trivial way, resulting in different gradient directions in parameter space, and better performance with 1's than with 0's. (In the related context of restricted Boltzmann machines, the standard training technique by gradient ascent favors setting hidden units to 1 , for similar reasons OAAH11, Section 5].) This specific phenomenon disappears if, instead of the logistic function, the hyperbolic tangent is used as the activation function, or if the input is normalized. But this will not help if, for instance, the activities of internal units in a multilayer network are not centered on average. Scaling also has an effect on performance: for instance, a common recommendation [LBOM96] is to use $1.7159 \tanh (2 x / 3)$ instead of just $\tanh (x)$ as the activation function.

It would be interesting to have algorithms whose performance is insensitive to particular choices such as scaling factors in network construction, parameter encoding or data representation. We call an algorithm invariant, or intrinsic, if applying a change of variables to the parameters and activities results in the same learning trajectory. This is not the case for backpropagation (even after changing the learning rate): for instance, changing from 


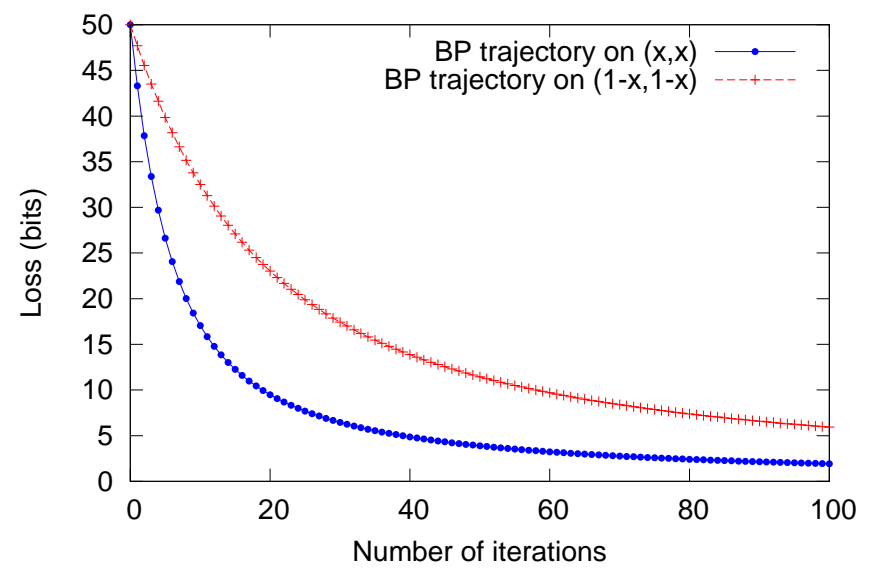

Figure 1: Backpropagation learns faster with 1's. A neural network with two layers of size 50 (no hidden layer) is trained to reproduce its input. A random binary sequence $x$ of length 50 with $75 \%$ of 1 's is generated. The network is trained on the input-output pair $(x, x)$ for 100 backpropagation steps (learning rate 0.01). The experiment is repeated on the input-output pair $(1-x, 1-x)$. In both cases all initial weights are set to 0 .

sigmoid to tanh activation amounts to dividing the connection weights by 4 and shifting the biases by half the weights (Eqs. (46)-(47)), which does not preserve gradient directions.

Invariance of an algorithm means fewer arbitrary design choices, and also more robustness: good performance on a particular problem indicates good performance over a whole class of problems equivalent to the first one by simple (e.g., affine) transformations.

Known invariant algorithms include Newton or quasi-Newton methods [BL88], or the natural gradient Ama98]. The latter, in particular, is invariant (and thus preserves performance) under a wide class of changes in the representation of the data and of the network, while Newton-like methods are only invariant under affine transforms. However, these methods are generally not scalable: the cost of maintaining the whole Hessian or Fisher information matrix is quadratic in parameter dimension and prohibitive for large networks BL88, LBOM96. The approximations made to ensure their scalability, such as keeping only diagonal terms [LBOM96, SZL13, making small-rank approximations [RMB07], or using limited-memory BFGS (e.g. $\left[\mathrm{LNC}^{+} 11\right]$ for a recent example), break their invariance properties.

Scalable Riemannian methods for neural networks. In this work we introduce four invariant algorithms adapted to four different scalability constraints. For this we develop a suitable theoretical framework in which 
to build invariant algorithms, by treating neural networks in the context of Riemannian geometry.

For a network with $n$ units, $n_{\text {out }}$ output units and at most $d$ incoming connections per unit, processing each data sample with backpropagation has an algorithmic cost $O(n d)$. The most lightweight of our invariant algorithms has $O(n d)$ cost per sample as well. The heaviest one is more faithful to the true natural gradient but has a cost $O\left(n d^{2}+n d n_{\text {out }}\right)$ and thus requires that the network is sparsely connected (the average number of units influencing a given unit must not be too large) and that the output layer is not too large. This latter condition is typically fulfilled in classification tasks.

The unitwise natural gradient is a scaled-down version of Amari's natural gradient Ama98 in which the blocks of incoming parameters to each unit are treated independently, thus dealing, at each unit, with a square matrix indexed by the incoming parameters to this unit. This has been proposed as far back as Kur94 to train neural networks; however the algorithm in Kur94 is limited to networks with only one hidden layer, because it relies on an explicit symbolic computation of entries of the Fisher matrix. Here Proposition 27 allows for an efficient computation of the exact Fisher information matrix in arbitrary neural networks, by doing $n_{\text {out }}$ distinct backpropagations for each sample in the dataset. As a result, the unitwise natural gradient is adapted to situations where both the connectivity of the network and the output layer are reasonably small: the algorithmic cost of processing each data sample is $O\left(n d^{2}+n d n_{\text {out }}\right)$.

The backpropagated metric gradient can be described as a blockwise quasi-Newton method in which several approximations (Gauss-Newton and neglecting cross-unit terms) are used. However, we describe it in an intrinsic way: it stems from a well-defined backpropagated metric on parameter space, in which no approximations are involved. Invariance properties follow from this viewpoint. It is adapted to networks with reasonably small connectivity but output layers of arbitrary size: the cost of processing a data sample is $O\left(n d^{2}\right)$.

The quasi-diagonal natural gradient and quasi-diagonal backpropagated metric gradient apply a "quasi-diagonal reduction" to these two algorithms, which removes the quadratic dependency on connectivity at each unit. This is done in a specific way to keep some (but not all) of the invariance properties, such as insensitivity to using sigmoid or $1.7159 \tanh (2 x / 3)$. The quasi-diagonal natural gradient still requires that the output layer is not too large, with a cost of $O\left(n d n_{\text {out }}\right)$ per data sample, whereas the quasidiagonal backpropagated metric gradient has the same $O(n d)$ complexity as ordinary backpropagation. These quasi-diagonal methods have not been described before, to the best of our knowledge.

In this context, we also clarify another method found in the literature APF00, RMB07. It is related to, and sometimes confused with, the natural gradient (discussion in [PB13]). We call this method the outer product 
metric gradient and introduce a scaled-down, invariant version. We prove a novel interpretation of this method as the unique one that, at each step, spreads the improvement most uniformly over all training samples (Proposition [15).

Organization of the text. In Section 1 we give the explicit form of the algorithms, without justification, to serve as a reference for implementation. In Section 2 we provide the mathematical principles behind the algorithms, starting with the relationship between gradients, metrics, and choice of coordinates (Section 2.1), then using the tools of Riemannian geometry to build several invariant metrics for neural networks (Section 2.2 and Appendix C) together with a way of computing them. In Section 2.3 we introduce the quasi-diagonal reduction of a metric. These metrics produce associated gradient descents (Section 2.4). In Section 2.5 we discuss in detail the case of the Fisher metric for neural networks and various ways to compute or approximate it. In Section 3 we present some mathematical properties of these algorithms, focusing on invariance by change of coordinates (Section 3.2) and a "best-fit" interpretation (Section 3.3). Section 4 contains a set of small-scale experiments as a proof of concept for the new algorithms.

A companion article Oll13 develops related ideas for recurrent neural networks and provides more in-depth experiments on complex symbolic data sequences.

We now provide an introduction to how invariant algorithms are built, and an overview of the experimental results.

Gradient descents and metrics. To build these invariant algorithms, we use gradient descent in suitable invariant metrics.

Backpropagation is the simple gradient descent over parameter space. Gradient descents follow the steepest direction of change in parameter space, and implicitly rely on a norm (or quadratic form, or metric) to define the steepest direction: the gradient step $x \leftarrow x-\eta \nabla f$ can also be rewritten (for small enough $\eta$, up to $O\left(\eta^{2}\right)$ and for regular enough functions $f$ ) as

$$
x \leftarrow \underset{y}{\arg \min }\left\{f(y)+\frac{1}{2 \eta}\|y-x\|^{2}\right\}
$$

namely, the gradient descent moves into the direction yielding the smallest values of $f$, penalized by the distance from the current point, measured by the square norm $\|y-x\|^{2}$. For backpropagation this norm $\|\cdot\|^{2}$ is the numerical change in the values of the parameters: backpropagation provides the direction of largest improvement for a minimal change in these numerical values. Hence simple changes in parametrization influence the behavior of the algorithm. On the other hand, norms $\|\cdot\|^{2}$ based on what the network 
does, rather than how it is represented as numbers, will lead to "intrinsic" algorithms. This is one of the ideas behind Amari's natural gradient.

In Section 2 we build several invariant norms, by placing neural networks in the context of differential manifolds and Riemannian geometry. The gradient descent coming from an invariant norm (Riemannian metric) will itself be invariant. Moreover, any gradient descent using any norm has the property that small enough learning rates ensure performance improvement at each step.

The resulting algorithms are all invariant under a number of transformations, including affine reparametrization of the unit activities. Among the invariance properties enjoyed by the unitwise natural gradient and the backpropagated metric (but not their quasi-diagonal reductions) are linear recombinations of the input received by a given unit in the network, so that a unit receiving signals $f$ and $f+\varepsilon g$ (as functions over the dataset) from incoming units will learn an output correlated to $g$ just as fast as a unit receiving signals $f$ and $g$ (on the input layer this can be accounted for by normalizing and de-correlating the inputs, but this could occur at internal units as well). Thus these gradients have a "best-fit" interpretation (Section 3.3): at each unit they solve a least-square problem of matching input signals and desired backpropagated output, an interpretation proposed in Kur94.

The quasi-diagonal reductions of these algorithms are based on the observation that there is a distinction between weights $w_{i k}$ and $w_{j k}$ coming to $k$ from different units, but no intrinsic mathematical separation between weights and biases. Intuitively, given that unit $k$ receives a signal $w_{i k} a_{i}$ from unit $i$, if we change $w_{i k}$ to $w_{i k}+\delta w_{i k}$, the average signal to unit $k$ will change by $\delta w_{i k} \bar{a}_{i}$ where $\bar{a}_{i}$ is the average activation of $i$. Hence it might be a good idea to automatically add $-\delta w_{i k} \bar{a}_{i}$ to the bias of $k$, to compensate. The quasi-diagonal algorithms we present are more sophisticated versions of this, tuned for invariance and using weighted averages. The few added terms in the update sometimes greatly improve performance (Fig 5 on page 50).

Arguably, none of these algorithms is second-order: the update on parameter $\theta$ takes the form $\theta \leftarrow \theta-A(\theta)^{-1} \partial_{\theta} f$ where $A(\theta)$ is a matrix depending on the network but not on the objective function $f$. This matrix comes from (Riemannian) metrics evaluating the magnitude of the effect on the output of changes in a given direction, thus providing a suitable learning rate for each direction, without estimating derivatives of gradients. Second-order effects are emulated in the same way the Gauss-Newton algorithm emulates the Newton method1.

\footnotetext{
${ }^{1}$ Actually, in the framework of differential geometry, without a metric, the Hessian is only defined at local optima of the function [GHL87, paragraph 3.37], so one could say that in such a setting the Newton method approximates the Gauss-Newton algorithm rather than the other way around.
} 


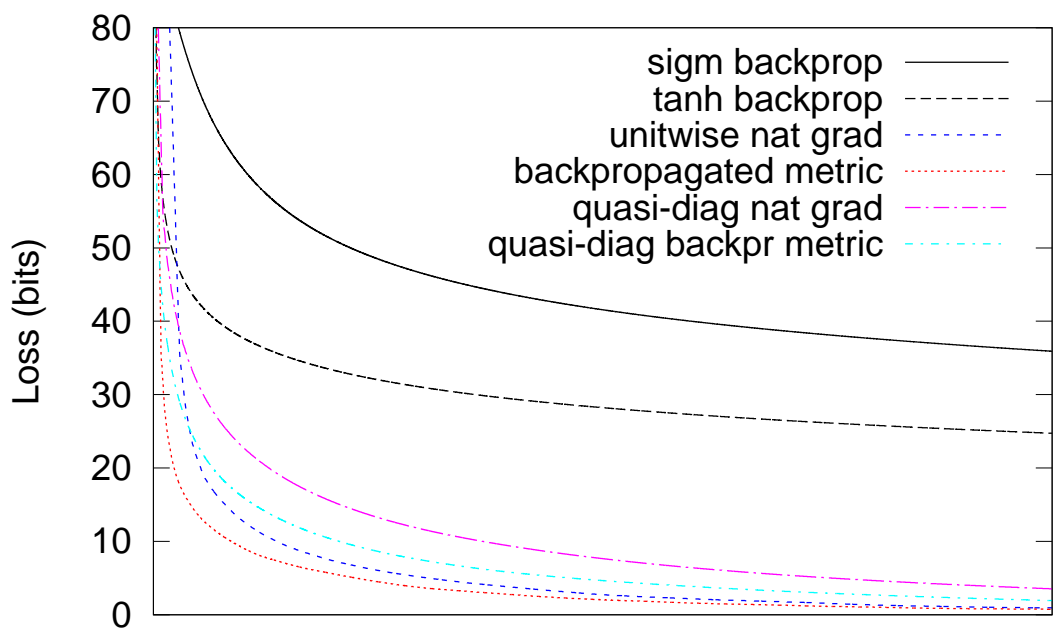

Computation time (arbitrary units)

Figure 2: Auto-encoding using a 100-30-10-30-100 deep sparsely connected network. Comparison of backpropagation using sigmoid and tanh activation, and the four algorithms described in Section 1, for a given computation time budget.

Experimental proof of concept. While the focus of this article is mainly the mathematics of neural network training, we quickly tested experimentally the implementability and impact of using the various methods. We selected a very simple auto-encoding problem on which we expected that any training method would perform well. A sparsely connected network with 5 layers of size $100,30,10,30$, and 100 was built, and 16 random length-100 binary strings were fed to the input layer, with the target equal to the input. Ideally the network learns to encode each of the 16 samples using 4 bits on the middle layer (thus with room to spare) and rewrites the output from this. The details are given in Section 4 .

Even then, backpropagation performs poorly: after 10,000 batch passes the average log-loss is about 36 bits per sample (out of 100) for sigmoid backpropagation, and about 25 bits per sample for tanh backpropagation. Note that 30 bits per sample would correspond to a method which learns only the parameters of the output layer and can reproduce the output if someone fills the last hidden layer with the correct 30 bits.

For the same total computation time equivalent to 10,000 batch backpropagation 2 , the quasi-diagonal algorithms have a log loss of about 1.5 to

\footnotetext{
${ }^{2}$ as measured in CPU time on a personal computer, but this can depend a lot on implementation details
} 
3.5 bits per sample, and both the unitwise natural gradient and the backpropagated metric gradient have a log loss of 0.3 to 1.5 bit per sample, thus essentially solving the problem. See Figure 2.

The impact of the few non-diagonal terms in the quasi-diagonal algorithms was tested by removing them. In this case the quasi-diagonal backpropagated metric gradient reduces to the diagonal Gauss-Newton method ([LBOM96, Section 7.4], also used in [SZL13]). This breaks the invariance properties, thus the impact is different for sigmoid or tanh implementations. The diagonal Gauss-Newton method in sigmoid implementation was found to perform more poorly, with a final log-loss of about 12 bits per sample (Figure 5 on page 50), while in tanh implementation it comes somewhat close to the quasi-diagonal algorithms at about 3.5 bits per sample (presumably because in our problem, the activity of all units, not only input units, stay perfectly centered during training). Thus the quasi-diagonal backpropagated metric gradient can be seen as "the invariant way" to write the diagonal Gauss-Newton method, while performance of the latter is not at all invariant.

We also compared the exact unitwise natural gradient obtained thanks to Proposition 27, to a variant of the natural gradient in which only the gradient terms $b b^{\top}$ corresponding to the target for each sample are added to the Fisher matrix ([APF00, RMB07] and Section [2.5 below). The latter, when implemented unitwise, performs rather poorly on this auto-encoding task, with a log loss of about 25 to 28 bits per sample. The reason, discussed in Section 4, may be that quality of this approximation to the Fisher matrix strongly depends on output dimensionality.

One lesson from the numerical experiments is that the regularization term $\varepsilon$ Id added to the matrices, needed to prevent bad behavior upon inversion, formally breaks the invariance properties: individual trajectories in sigmoid or tanh implementations, initialized in the same way, start to differ after a dozen iterations. Still, overall performance is not affected and is the same in both implementations (Figure 4, p. 48).

Though the quasi-diagonal methods perform well, the only methods to sometimes reach very small values of the loss function on this example (less than 0.1 bit per sample) are the unitwise natural gradient and the backpropagated metric, which at each unit maintain a full matrix over the incoming parameters and thus achieve invariance under affine recombination of incoming signals. These two methods are relevant only when network connectivity is not too high. This highlights the interest of sparsely connected networks from a theoretical viewpoint.

\section{Notation for neural networks}

Consider a directed neural network model: a set $\mathcal{L}$ of units together with a set of directed edges $i \rightarrow j$ for $i, j \in \mathcal{L}$, without cycle. Let $\mathcal{L}_{\text {out }}$ be the 
output units, that is, the units with no outgoing edges 3 , and similarly let $\mathcal{L}_{\text {in }}$ be the set of units without incoming edges. Let $s: \mathbb{R} \rightarrow \mathbb{R}$ be an activation function. Given an activation level for the input units, each unit $j$ gets an activation level

$$
a_{j}=s\left(\sum_{i \rightarrow j} w_{i j} a_{i}\right)
$$

depending on the activation levels of the units $i$ pointing to $j$ and on the firing coefficients $w_{i j}$ from $i$ to $j$. Biases are treated as the weights $w_{0 j}$ from a special always-activated unit $0\left(a_{0} \equiv 1\right)$ connected to every other unit. A common choice for the activation function $s$ is the logistic [RN03] function $s(V)=\frac{\mathrm{e}^{V}}{1+\mathrm{e}^{V}}=\frac{1}{1+\mathrm{e}^{-V}}$, although for instance [LBOM96] recommends the hyperbolic tangent $s(V)=\tanh (V)$, related to the logistic by $\tanh (V)=$ $2\left(\frac{1}{1+\mathrm{e}^{-2 V}}\right)-1$. We refer to [RN03, which we mostly follow with minor changes in notation.

For a given non-input unit $j$, we call the parameters $w_{0 j}$ and $w_{i j}$ for $i \rightarrow j$ the set of incoming parameters to unit $j$.

The dataset for this network is a set $\mathcal{D}$ of inputs, where each input $x \in \mathbb{R}^{\mathcal{L}_{\text {in }}}$ is a real-valued vector over the input layer. For each input is given a target $y$ in an arbitrary space. We view the network as a probabilistic generative model: given an input $a_{i}=x_{i}$ on the input layer $\mathcal{L}_{\text {in }}$, we assume that the activations of the output layer are interpreted in a fixed way as a probability distribution $\omega(y)$ over the target space. The goal is to maximize the probability to output $y$ on input $x$ : we define the loss function

$$
\ell(\omega, y):=-\ln \omega(y)
$$

the sum of which over the dataset is to be minimized. For instance, interpreting the output layer activities $\left(a_{k}\right)_{k \in \mathcal{L}_{\text {out }}}$ as Gaussian variables with mean $a_{k}$ and variance 1 leads to a quadratic loss function $\ell$.

EXAmple 1 (Square-loss, Bernoulli, AND Two ClassificaTION INTERPRETATIONS). The square-loss interpretation of the output layer sends the activations $\left(a_{k}\right)_{k \in \mathcal{L}_{\text {out }}}$ of the output layer to a random variable $Y=\left(Y_{k}\right)_{k \in \mathcal{L}_{\text {out }}}$ of independent Gaussian variables, where each $Y_{k} \sim \mathcal{N}\left(a_{k}, 1\right)$ is a Gaussian of mean $a_{k}$.

Assume that the activities $a_{i}$ of units in the network lie in $[0 ; 1]$. The Bernoulli interpretation of the output layer is a Bernoulli distribution as follows: given the activations $\left(a_{k}\right)_{k \in \mathcal{L}_{\text {out }}}$ of the output layer, the final out-

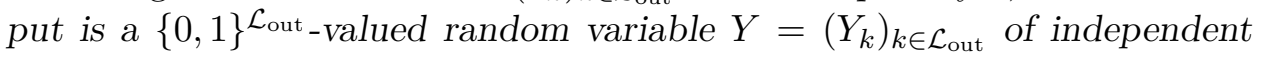
Bernoulli variables, where the activations $a_{k}$ give the probability to have $Y_{k}=1$, namely $\operatorname{Pr}\left(Y_{k}=1\right)=a_{k}$.

\footnotetext{
${ }^{3}$ This restriction on output units is not necessary (any unit could be an output unit) but simplifies notation.

${ }^{4}$ What is $w_{i j}$ for some authors is $w_{j i}$ for others. Our convention is the same as RN03] but for instance LBOM96 follows the opposite convention.
} 
For classification, the interpretation must send the output activations $\left(a_{k}\right)_{k \in \mathcal{L}_{\text {out }}}$ to a probability distribution over the indices $k$ in the output layer. In the softmax interpretation $\sqrt[5]{5}$, the probability of class $k \in \mathcal{L}_{\text {out }}$ is $\mathrm{e}^{a_{k}} / \sum_{k^{\prime} \in \mathcal{L}_{\text {out }}} \mathrm{e}^{a_{k^{\prime}}}$. In the spherical interpretation 6 , the probability of class $k \in \mathcal{L}_{\text {out }}$ is $a_{k}^{2} /\left(\sum_{k^{\prime} \in \mathcal{L}_{\text {out }}} a_{k^{\prime}}^{2}\right)$.

Remark 16 covers the case when the interpretation depends on extra parameters $\vartheta$, such as a softmax $\operatorname{Pr}(k)=\mathrm{e}^{\vartheta_{k} a_{k}} / \sum_{k^{\prime} \in \mathcal{L}_{\text {out }}} \mathrm{e}^{\vartheta_{k^{\prime}} a_{k^{\prime}}}$ with trainable weights $\vartheta_{k}$.

Backpropagation. A common way to train the network on a given target value $y$ is by backpropagation, which amounts to a gradient descent over the parameters $w_{i j}$. For a given loss function, define the backpropagated value $b_{i}$ at each unit $i$ by

$$
b_{i}:=-\frac{\partial \ell}{\partial a_{i}}
$$

so that $b_{i}$ indicates how we should modify the activities to decrease $\ell$. Then the value of $b_{i}$ satisfy the backpropagation equations [RN03] from the output layer:

$$
b_{i}=\sum_{j, i \rightarrow j} w_{i j} r_{j} b_{j} \quad \text { for } i \notin \mathcal{L}_{\text {out }}
$$

where the activation levels $a_{i}$ have first been computed by forward propagation, and where let $r_{j}$ stand for the value of the derivative of the activation function at unit $j$ :

$$
r_{j}:=s^{\prime}\left(\sum_{i \rightarrow j} w_{i j} a_{i}\right)= \begin{cases}a_{j}\left(1-a_{j}\right) & \text { for sigmoid activation function } \\ 1-a_{j}^{2} & \text { for tanh activation function }\end{cases}
$$

The backpropagated values on the output layer are computed directly by (44), for instance,

$$
b_{i}= \begin{cases}y_{i}-a_{i} & \text { (square-loss interpretation) } \\ \frac{y_{i}-a_{i}}{a_{i}\left(1-a_{i}\right)} & \text { (Bernoulli interpretation) } \\ y_{i}-\frac{\mathrm{e}^{a_{i}}}{\sum_{k \in \mathcal{L}_{\text {out }}} \mathrm{e}^{a_{k}}} & \text { (softmax interpretation) } \\ \frac{2 y_{i}}{a_{i}-\frac{2 a_{i}}{\sum_{k \in \mathcal{L}_{\text {out }}} a_{k}^{2}}} & \text { (spherical interpretation) }\end{cases}
$$

for $i \in \mathcal{L}_{\text {out }}$.

\footnotetext{
${ }^{5}$ Usually combined with a linear activation function $(s=\mathrm{Id})$ on the last layer

${ }^{6}$ This latter example is motivated by a theoretical argument: the set of probability distributions over a finite set, equipped with its Fisher metric, is isometric to the positive quadrant in a sphere and so is naturally parametrized by numbers $a_{k}$ with $\sum a_{k}^{2}=1$, and these variables yield a slightly simpler expression for the Fisher matrix. Besides, taking squares gives a boost to the most activated output unit, in a smooth way, as in the softmax interpretation.
} 
The backpropagated values are used to compute the gradient of the loss function with respect to the parameters $w_{i j}$. Indeed we have

$$
\frac{\partial \ell}{\partial w_{i j}}=-a_{i} r_{j} b_{j}
$$

for each edge $(i j)$ in the network. (This includes the bias $w_{0 j}$ using $a_{0} \equiv 1$.)

It is sometimes more convenient to work with the reduced variables

$$
\tilde{b}_{i}:=r_{i} b_{i}
$$

which satisfy the backpropagation equation

$$
\tilde{b}_{i}=r_{i} \sum_{j, i \rightarrow j} w_{i j} \tilde{b}_{j} \quad \text { for } i \notin \mathcal{L}_{\text {out }}
$$

and

$$
\frac{\partial \ell}{\partial w_{i j}}=-a_{i} \tilde{b}_{j}
$$

The gradient descent with learning rate $\eta>0$ is then defined as the following update on the firing coefficients:

$$
w_{i j} \leftarrow w_{i j}-\eta \frac{\partial \ell}{\partial w_{i j}}=w_{i j}+\eta a_{i} r_{j} b_{j}=w_{i j}+\eta a_{i} \tilde{b}_{j}
$$

\section{Four invariant gradient algorithms}

We now describe four gradient algorithms for network training: the unitwise natural gradient, the quasi-diagonal natural gradient, the backpropagated metric gradient, and the quasi-diagonal backpropagated metric gradient. Each of these algorithms is adapted to a different scalability constraint. The unitwise natural gradient requires low connectivity and a small output layer; the quasi-diagonal natural gradient requires a small output layer; the backpropagated metric gradient requires low connectivity; the quasidiagonal backpropagated metric gradient has the same asymptotic complexity as backpropagation.

These algorithms are the implementation of the more general versions described in Section 2, As they are designed for invariance properties, implementing them using either sigmoid or tanh activation function should result in the same output, learning trajectories, and performance, provided the initialization is changed accordingly (Section 3.2). However, the Bernoulli and classification interpretations of the output layer assumes that the activities lie in $[0 ; 1]$, as in sigmoid activation.

We first present the algorithms in a batch version. It is straightforward to adapt them to use random mini-batches from the dataset. In Section 1.3 they are also adapted to an online setting: this can be done using standard techniques because the main quantities involved take the form of averages over the dataset, which can be updated online. 


\subsection{Unitwise natural gradient and quasi-diagonal natural gra- dient}

The unitwise natural gradient has been proposed as far back as Kur94 to train neural networks; however the presentation in Kur94 is limited to networks with only one hidden layer, because it relies on an explicit symbolic computation of entries of the Fisher matrix. Proposition [27] below allows for an efficient computation of the exact Fisher information matrix by doing $n_{\text {out }}$ distinct backpropagations for each sample in the dataset. This relies on linearity of backpropagation, as follows.

Definition 2 (BACKPropagation transfer rates). Fix an input $x$ for the network and compute the activities by forward propagation. Let $k$ be a unit in the network and $k_{\text {out }}$ be a unit in the output layer. Define the backpropagation transfer rates $J_{k}^{k_{\text {out }}}$ from $k_{\text {out }}$ to $k$ by backpropagating the value 1 at $k_{\text {out }}$. Formally:

$$
\begin{cases}J_{k_{\text {out }}}^{k_{\text {out }}}:=1, \quad J_{k}^{k_{\text {out }}}:=0, & \text { for } k \neq k_{\text {out }} \text { in the output layer } \mathcal{L}_{\text {out }} \\ J_{k}^{k_{\text {out }}}:=\sum_{j, k \rightarrow j} w_{k j} r_{j} J_{j}^{k_{\text {out }}} & \text { for non-output units } k\end{cases}
$$

where $r_{j}$ is the derivative of the activation function, given by (6).

These transfer rates have the property that if backpropagation values $b$ are set on the output layer, then $b_{k}=\sum_{k_{\text {out }} \in \mathcal{L}_{\text {out }}} J_{k}^{k_{\text {out }}} b_{k_{\text {out }}}$ for any unit $k$ (see also [LBOM96, Section 7.2]).

Computation of the transfer rates can be done by $n_{\text {out }}$ distinct backpropagations. There are further simplifications, since the transfer rates for $k$ in the input layer are never used (as there are no incoming parameters), and the transfer rates on the last hidden layer are readily computed as $J_{k}^{k_{\text {out }}}=w_{k k_{\text {out }}} r_{k_{\text {out }}}$. Thus it is enough to backpropagate the transfer rates from the last hidden layer to the first hidden layer. In particular, with only one hidden layer (the case considered in Kur94 for the Fisher matrix) no backpropagation is needed.

Definition 3 (Fisher modulus). Fix an input $x$ for the network and compute the activities by forward propagation. For each unit $k$ in the network, define the Fisher modulus $\Phi_{k}(x)$ of unit $k$ on input $x$ as follows, depending on output layer interpretation.

- For the Bernoulli interpretation, set

$$
\Phi_{k}(x):=\sum_{k_{\text {out }} \in \mathcal{L}_{\text {out }}} \frac{\left(J_{k}^{k_{\text {out }}}\right)^{2}}{a_{k_{\text {out }}}\left(1-a_{k_{\text {out }}}\right)}
$$

- For the square-loss interpretation, set

$$
\Phi_{k}(x):=\sum_{k_{\text {out }} \in \mathcal{L}_{\text {out }}}\left(J_{k}^{k_{\text {out }}}\right)^{2}
$$


- For the softmax interpretation, set

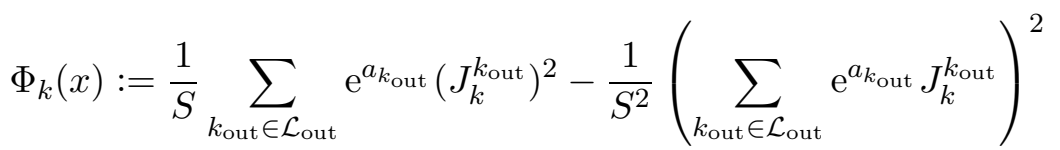

where $S:=\sum_{k_{\text {out }} \in \mathcal{L}_{\text {out }}} \mathrm{e}^{a_{k_{\text {out }}}}$.

- For the spherical interpretation, set

$$
\Phi_{k}(x):=\frac{4}{S} \sum_{k_{\text {out }} \in \mathcal{L}_{\text {out }}}\left(J_{k}^{k_{\text {out }}}\right)^{2}-\frac{4}{S^{2}}\left(\sum_{k_{\text {out }} \in \mathcal{L}_{\text {out }}} a_{k_{\text {out }}} J_{k}^{k_{\text {out }}}\right)^{2}
$$

where $S:=\sum_{k_{\text {out }} \in \mathcal{L}_{\text {out }}} a_{k_{\text {out }}}^{2}$.

Definition 4 (UNitwise Fisher matrix). Let $k$ be a unit in the network. Let $E_{k}$ be the set of incoming units to $k$ (including the alwaysactivated unit 0$)$. The unitwise Fisher matrix at unit $k$ is the $\left(\# E_{k}\right) \times\left(\# E_{k}\right)$ matrix $F^{(k)}$ defined by

$$
F_{i j}^{(k)}:=\mathbb{E}_{x \in \mathcal{D}} a_{i} a_{j} r_{k}^{2} \Phi_{k}
$$

for $i$ and $j$ in $E_{k}$ (including the unit 0 with $a_{0} \equiv 1$ ), with $\Phi_{k}$ the Fisher modulus of Definition 3. Here $\mathbb{E}_{x \in \mathcal{D}}$ represents the average over samples $x$ in the dataset (all the terms $a_{i}, a_{j}, r_{k}, \Phi_{k}$ depend on the input to the network).

By Proposition 27 below, $F_{i j}^{(k)}$ is the block of the Fisher information matrix associated with the incoming parameters to $k$, hence the name.

Definition 5 (Unitwise NATURAL GRADIENT). The unitwise natural gradient with learning rate $\eta>0$ updates the parameters of the network as follows.

For each unit $k$, define the vector $G^{(k)}$ by

$$
G_{i}^{(k)}:=-\mathbb{E}_{x \in \mathcal{D}} \frac{\partial \ell(y)}{\partial w_{i k}}=\mathbb{E}_{x \in \mathcal{D}} a_{i} r_{k} b_{k}
$$

where $b_{k}$ is the backpropagated value at $k$ obtained from the target $y$ associated with $x$, and where $i$ runs over the incoming units to $k$ (including the always-activated unit $i=0$ ). Compute the vector

$$
\delta w^{(k)}:=\left(F^{(k)}\right)^{-1} G^{(k)}
$$

Then the parameters of the network are updated by

$$
w_{i k} \leftarrow w_{i k}+\eta \delta w_{i}^{(k)}
$$


The asymptotic algorithmic cost of the unitwise natural gradient is as follows. Computing $\tau$ requires $n_{\text {out }}$ distinct backpropagations for each input $x$. For a network with $n$ units, $n_{\text {out }}$ output units, and at most $d$ incoming connections per unit, this costs $O\left(n_{\text {out }} n d\right)$ per data sample (this can be reduced in some cases, as discussed above). Computing $\Phi$ takes $O\left(n n_{\text {out }}\right)$. Computing $F_{i j}^{(k)}$ for every $k$ takes $O\left(n d^{2}\right)$. Computing the gradient requires inverting these matrices, which takes $O\left(n d^{3}\right)$ but is done only once in each (mini-)batch, thus if the size of batches is larger than $d$ this cost is negligible; if the size of batches is smaller than $d$ or in an online setting, inversion can be done recursively using the Sherman-Morrison formula at a cost of $O\left(n d^{2}\right)$ per data sample. So the overall cost of this gradient is $O\left(n d^{2}+n_{\text {out }} n d\right)$ per data sample.

This algorithmic cost is fine if connectivity is low. We now define a more light-weight version in case connectivity is large. Its computational cost is equivalent to that of ordinary backpropagation provided the output layer is small.

DeFinition 6 (QuASI-Diagonal NATURAL GRADIENT). The quasidiagonal natural gradient with learning rate $\eta>0$ updates the parameters of the network as follows.

For each unit $k$, compute only the entries $F_{00}^{(k)}, F_{0 i}^{(k)}$, and $F_{i i}^{(k)}$ of the unitwise Fisher matrix at $k$. Define the vector $G^{(k)}$ as in (19) above. Define the vector $\delta w^{(k)}$ by

$$
\begin{aligned}
& \delta w_{i}^{(k)}=\frac{G_{i}^{(k)} F_{00}^{(k)}-G_{0}^{(k)} F_{0 i}^{(k)}}{F_{i i}^{(k)} F_{00}^{(k)}-\left(F_{0 i}^{(k)}\right)^{2}} \quad \text { for } i \neq 0 \\
& \delta w_{0}^{(k)}=\frac{G_{0}^{(k)}}{F_{00}^{(k)}}-\sum_{i \neq 0} \frac{F_{0 i}^{(k)}}{F_{00}^{(k)}} \delta w_{i}^{(k)}
\end{aligned}
$$

and update the parameters of the network by

$$
w_{i k} \leftarrow w_{i k}+\eta \delta w_{i}^{(k)}
$$

With respect to the unitwise natural gradient, the algorithmic cost does not involve any $O\left(n d^{2}\right)$ terms because we only compute $O(d)$ entries of the matrices $F$ and do not require a matrix inversion. The variables $\tau$ and $\Phi$ still need to be computed. Thus the overall complexity is reduced to $O\left(n_{\text {out }} n d\right)$.

The quasi-diagonal formulas may seem arbitrary. If we remember that (omitting the superscript $(k)) F_{00}=\mathbb{E}_{x \in \mathcal{D}} r_{k}^{2} \Phi_{k}, F_{0 i}=\mathbb{E}_{x \in \mathcal{D}} a_{i} r_{k}^{2} \Phi_{k}$, and $F_{i i}=\mathbb{E}_{x \in \mathcal{D}} a_{i}^{2} r_{k}^{2} \Phi_{k}$, we can consider these sums as expectations over the dataset with weights $r_{k}^{2} \Phi_{k}$. Then the weighted average of $a_{i}$ is $A_{i}=F_{0 i} / F_{00}$ and its weighted variance is $V_{i}=F_{i i} / F_{00}-A_{i}^{2}$ so that we have

$$
\delta w_{i}=\frac{G_{i}-G_{0} A_{i}}{F_{00} V_{i}}
$$


and in particular the denominator is always positive unless the activity of unit $i$ is constant (in this case, the numerator vanishes too).

A possible interpretation is as follows: If the activity $a_{i}$ of unit $i$ is not centered on average over the dataset (with the weights above), increasing a weight $w_{i k}$ not only increases the influence of unit $i$ over unit $k$, but also shifts the average activity of unit $k$, which may not be desirable. Using the method above, if $a_{i}$ is not centered, when we change $w_{i k}$ a corresponding term is automatically subtracted from the bias $w_{0 k}$ so as not to shift the average activity of unit $k$, as discussed in the introduction. On the other hand if the activity $a_{i}$ is centered, then the update is diagonal, and scaled by the inverse "variance" $1 / V_{i}$.

\subsection{Backpropagated metric gradient and quasi-diagonal back- propagated metric gradient}

Computing the Fisher matrix as above requires performing $n_{\text {out }}$ backpropagations for each sample. If one tries to compute the Fisher modulus $\Phi$ directly by backpropagation, the backpropagation equation involves crossterms between different units. Neglecting these cross-terms results in a simpler version of the Fisher modulus which can be computed in one backward pass; the corresponding backpropagation equation is well-known as an approximation of the Hessian [LBOM96, Section 7]. It turns out this quantity and the associated metric are still intrinsic.

Definition 7 (BaCkPropagated modulus). Fix an input $x$ for the network and compute the activities by forward propagation. Define the backpropagated modulus $m_{k}(x)$ for each unit $k$ by

$$
m_{k}(x):=\sum_{j, k \rightarrow j} w_{k j}^{2} r_{j}^{2} m_{j}(x)
$$

if $k$ is not an output unit, and, depending on output interpretation,

$$
m_{k}(x):= \begin{cases}\frac{1}{a_{k}\left(1-a_{k}\right)} & (\text { Bernoulli) } \\ 1 & (\text { square-loss }) \\ \frac{\mathrm{e}^{a_{k}}}{S}\left(1-\frac{\mathrm{e}^{a_{k}}}{S}\right), \quad S=\sum_{k_{\text {out }} \in \mathcal{L}_{\text {out }}} \mathrm{e}^{a_{k_{\text {out }}}} & (\text { softmax }) \\ \frac{4}{S}\left(1-\frac{a_{k}^{2}}{S}\right), \quad S=\sum_{k_{\text {out }} \in \mathcal{L}_{\text {out }}} a_{k_{\text {out }}}^{2} & (\text { spherical) }\end{cases}
$$

for $k$ in the output layer.

Definition 8 (BACKPRopagated Metric). Let $k$ be a unit in the network. Let $E_{k}$ be the set of incoming units to $k$ (including the alwaysactivated unit 0$)$. The backpropagated metric at unit $k$ is the $\left(\# E_{k}\right) \times\left(\# E_{k}\right)$ matrix $M^{(k)}$ defined by

$$
M_{i j}^{(k)}:=\mathbb{E}_{x \in \mathcal{D}} a_{i} a_{j} r_{k}^{2} m_{k}
$$


for $i$ and $j$ in $E_{k}$ (including the unit 0 with $a_{0} \equiv 1$ ). Here $\mathbb{E}_{x \in \mathcal{D}}$ represents the average over samples $x$ in the dataset (all the terms $a_{i}, a_{j}, a_{k}, m_{k}$ depend on the input to the network).

The backpropagated metric gradient can thus be described as an approximate, blockwise Hessian method in which the Hessian is approximated by the Gauss-Newton technique with, in addition, cross-unit terms neglected. Such a method turns out to be intrinsic.

DEFINITION 9 (BACKPROPAGATED METRIC GRADIENT). The backpropagated metric gradient with learning rate $\eta>0$ updates the parameters of the network as follows.

For each unit $k$, define the vector $G^{(k)}$ by

$$
G_{i}^{(k)}:=-\mathbb{E}_{x \in \mathcal{D}} \frac{\partial \ell(y)}{\partial w_{i k}}=\mathbb{E}_{x \in \mathcal{D}} a_{i} r_{k} b_{k}
$$

where $b_{k}$ is the backpropagated value at $k$ obtained from the target $y$ associated with $x$, and where $i$ runs over the incoming units to $k$ (including the always-activated unit $i=0$ ). Compute the vector

$$
\delta w^{(k)}:=\left(M^{(k)}\right)^{-1} G^{(k)}
$$

Then the parameters of the network are updated by

$$
w_{i k} \leftarrow w_{i k}+\eta \delta w_{i}^{(k)}
$$

The algorithmic cost of the backpropagated metric gradient is $O\left(n d^{2}\right)$ per data sample, with notation as above. Indeed, computing $m$ costs the same as a backpropagation pass, namely $O(n d)$. Computing the matrices $M$ costs $O\left(n d^{2}\right)$. Inverting the matrices has no impact on the overall complexity, as explained after Definition 5. This cost is acceptable for small $d$ (sparsely connected networks). For large $d$ we define the following.

DEFINITION 10 (QUASI-DIAGONAL BACKPROPAGATED METRIC GRADIENT). The quasi-diagonal backpropagated metric gradient with learning rate $\eta>0$ updates the parameters of the network as follows.

For each unit $k$, compute only the entries $M_{00}^{(k)}, M_{0 i}^{(k)}$, and $M_{i i}^{(k)}$ of backpropagated metric at $k$. Define the vector $G^{(k)}$ as in (19) above. Define the vector $\delta w^{(k)}$ by

$$
\begin{aligned}
& \delta w_{i}^{(k)}=\frac{G_{i}^{(k)} M_{00}^{(k)}-G_{0}^{(k)} M_{0 i}^{(k)}}{M_{i i}^{(k)} M_{00}^{(k)}-\left(M_{0 i}^{(k)}\right)^{2}} \quad \text { for } i \neq 0 \\
& \delta w_{0}^{(k)}=\frac{G_{0}^{(k)}}{M_{00}^{(k)}}-\sum_{i \neq 0} \frac{M_{0 i}^{(k)}}{M_{00}^{(k)}} \delta w_{i}^{(k)}
\end{aligned}
$$

and update the parameters of the network by

$$
w_{i k} \leftarrow w_{i k}+\eta \delta w_{i}^{(k)}
$$


The same remarks as for the quasi-diagonal natural gradient apply for interpreting the various terms. The denominator $M_{i i}^{(k)} M_{00}^{(k)}-\left(M_{0 i}^{(k)}\right)^{2}$ can be seen as a weighted variance of the activity of unit $i$, and is positive unless $a_{i}$ is constant over the dataset. The contribution of $\delta w_{i}^{(k)}$ to $\delta w_{0}^{(k)}$ compensates the change of average activity induced by a change of $w_{i k}$.

The asymptotic cost of this update is $O(n d)$ per data sample, as for backpropagation.

If, in the quasi-diagonal backpropagated metric gradient, the non-diagonal terms are neglected $\left(M_{0 i}^{(k)}\right.$ is set to 0$)$, then this reduces to the diagonal Gauss-Newton method equations from [LBOM96, Section 7.4] (also used for instance in [SZL13]).

REMARK 11. On the incoming parameters to the output layer, the unitwise natural gradient and the backpropagated metric gradient coincide if the Bernoulli or square-loss interpretation is used. (Actually, with learning rate $\eta=1$ they also both coincide with the Newton method restricted to the output layer parameters.)

REMARK 12. Since these algorithms rely on inverting matrices, regularization is an issue. In practice, terms $\varepsilon$ Id have to be added to $F$ and $M$ before inversion; terms $\varepsilon$ have to be added to the diagonal terms $F_{00}^{(k)}, F_{i i}^{(k)}$, $M_{00}^{(k)}$ and $M_{i i}^{(k)}$ in the quasi-diagonal reduction. This formally breaks the invariance properties. Section 3.3 elaborates on this. Still, this operation preserves the guarantee of improvement for small enough learning rates.

\subsection{Adaptation to an online setting}

The unitwise natural gradient and unitwise backpropagated metric gradient both update the weights by

$$
\delta w=A^{-1} G
$$

with $G$ the gradient of the loss function over the dataset, and $A$ a positivedefinite, symmetric matrix. A key feature here is that the matrix $A$ takes the form of an expectation over the dataset: $F_{i j}^{(k)}=\mathbb{E}_{x \in \mathcal{D}} a_{i} a_{j} r_{k}^{2} \Phi_{k}$ for the Fisher matrix, and $M_{i j}^{(k)}=\mathbb{E}_{x \in \mathcal{D}} a_{i} a_{j} r_{k}^{2} m_{k}$ for the backpropagated metric.

Any such algorithm can be turned online using a standard construction as follows (compare e.g. [RMB07]). Another possibility is, of course, to use mini-batches.

In the following, $A$ stands for either the unitwise Fisher matrix or the backpropagated metric. Let $A(x)$ be the corresponding contribution of each input $x$ in the expectation, namely, $A(x)_{i j}^{(k)}=a_{i} a_{j} r_{k}^{2} \Phi_{k}$ for the Fisher metric and $A(x)_{i j}^{(k)}=a_{i} a_{j} r_{k}^{2} m_{k}$ for the backpropagated metric, so that $A=\mathbb{E}_{x \in \mathcal{D}} A(x)$. 
At each step $t$, we use one new sample in the dataset, update an estimate $A^{(t)}$ of $A$, and follow a gradient step for this sample, as follows.

- Initialize the matrix $A^{(0)}$ by using a small subsample $\mathcal{D}_{\text {init }} \subset \mathcal{D}$, for instance the first $n_{\text {init }}$ samples in the dataset:

$$
A^{(0)}:=\mathbb{E}_{x \in \mathcal{D}_{\text {init }}} A(x)
$$

- Fix a discount factor $0<\gamma<1$. For each new sample $x_{t}$, compute its contribution $A\left(x_{t}\right)$ and update $A$ by

$$
A^{(t)}:=(1-\gamma) A^{(t-1)}+\gamma A\left(x_{t}\right)
$$

- Compute the inverse of $A^{(t)}$ from the inverse of $A^{(t-1)}$ by the ShermanMorrison formula at each unit, using that $A\left(x_{t}\right)$ is a rank-one matrix at each unit. (This way matrix inversion is no more costly than the rest of the step.)

- Compute the negative gradient $G\left(x_{t}\right)$ of the loss function on input $x_{t}$ by backpropagation.

- Update the parameters by

$$
w \leftarrow w+\eta_{t}\left(A^{(t)}\right)^{-1} G\left(x_{t}\right)
$$

where $\eta_{t}$ is the learning rate.

- For the quasi-diagonal reductions of the algorithms, only the entries $A_{00}, A_{i i}$ and $A_{0 i}$ of the matrix $A$ are updated at each step. No matrix inversion is required for the update equations (22) $-(23)$ and (32)-(33).

We could also initialize $A^{(0)}$ to a simple matrix like Id, but this breaks the invariance properties of the algorithms.

The update rule for $A^{(t)}$ depends on the discount factor $\gamma$. It should be large enough so that a large number of data points contribute to the computation of $A$, but small enough to be reactive so that $A$ evolves as training gets along. In our setting, from the particular form of $A(x)$ at a unit $k$ we see that each $A\left(x_{t}\right)$ contributes a rank-one matrix. This means that $\gamma$ should be much smaller than $1 / n_{k}$ with $n_{k}$ the number of parameters at unit $k$, because otherwise the estimated matrix $A^{(t)}$ will be close to a low-rank matrix, and presumably a poor approximation of the true matrix $A$, unreliable for numerical inversion.

The same remark applies to the number $n_{\text {init }}$ of samples used for initialization: it should be somewhat larger than the number of parameters at each unit, otherwise $A^{(0)}$ will be of low rank. 


\section{Constructing invariant algorithms: Riemannian metrics for neural networks}

\subsection{Gradient descents and metrics, natural metrics}

The gradient of a function $f$ on $\mathbb{R}^{d}$ gives the direction of steepest ascent: among all (very small) vectors with a given norm, it provides the greatest variation of $f$. Formally, the gradient $\nabla f$ of a smooth function $f$ is defined by the property that

$$
f(x+\varepsilon v)=f(x)+\varepsilon\langle\nabla f, v\rangle+O\left(\varepsilon^{2}\right)
$$

for any vector $v$, for small enough $\varepsilon$. This depends on the choice of a scalar product $\langle\cdot, \cdot\rangle$. In an orthonormal basis, the coordinates of the gradient are simply the partial derivatives $\partial f / \partial x_{i}$ so that gradient descent is

$$
x_{i} \leftarrow x_{i}-\eta \partial f / \partial x_{i}
$$

in an orthonormal basis.

For a given norm of the vector $v$, the quantity $\langle\nabla f, v\rangle$ is maximal when $v$ is collinear with $\nabla f$ : so the gradient $\nabla f$ indeed gives the direction of steepest ascent among all vectors with a given norm. The gradient step $x \leftarrow x-\eta \nabla f$ can actually be rewritten (for small enough $\eta$, up to $O\left(\eta^{2}\right)$ and for regular enough functions $f$ ) as

$$
x \leftarrow \underset{y}{\arg \min }\left\{f(y)+\frac{1}{2 \eta}\|y-x\|^{2}\right\}
$$

namely, the gradient descent moves into the direction yielding the smallest values of $f$, penalized by the distance from the current point 7 . This makes it clear how the choice of the scalar product will influence the direction of the gradient $\nabla f$ : indeed, another scalar product will define another norm $\|v\|^{2}=\langle v, v\rangle$ for the vector $v$, so that the steepest direction among all vectors with a given norm will not be the same. The norm thus defines directions $v$ in which it is "cheap" or "expensive" to move; the gradient is the direction of steepest ascent taking this into account.

If we happen to work in a non-orthonormal basis of vectors $v_{1}, \ldots, v_{d}$, the gradient is given by $A^{-1} \partial f / \partial x$ where $\partial f / \partial x$ is the vector of partial derivatives with respect to the coordinates $x_{i}$ in the basis, and $A$ is the symmetric matrix made of the scalar products of the basis vectors with themselves: $A_{i j}:=\left\langle v_{i} \mid v_{j}\right\rangle$. Indeed, the change of variable $\tilde{x}:=A^{1 / 2} x$ provides an orthonormal basis, thus gradient descent for $\tilde{x}$ is $\tilde{x} \leftarrow \tilde{x}-$

\footnotetext{
${ }^{7}$ This can be used to define or study the direction of the gradient in more general metric spaces (e.g., AGS05, Chapter 2]).
} 
$\eta \partial f / \partial \tilde{x}=\tilde{x}-\eta A^{-1 / 2} \partial f / \partial x$. Thus, translating back on the variable $x$, the gradient descent of $f$ takes the form

$$
x \leftarrow x-\eta A^{-1} \partial f / \partial x
$$

Conversely, we can start with a norm on $\mathbb{R}^{d}$ defined through a positivedefinite, symmetric matrix $A$ (which thus defines "cheap" and "expensive" directions). The gradient descent using this norm will then be given by (42).

So any update of the form (42) above with $A$ a symmetric, positivedefinite matrix can be seen as the gradient descent of $f$ using some norm. The matrix $A$ may even depend on the current point $x$, defining a Riemannian metric in which the norm of a small change $\delta x$ of $x$ is

$$
\|\delta x\|^{2}:=\sum_{i j} \delta x_{i} A_{i j}(x) \delta x_{j}=\delta x^{\top} A(x) \delta x
$$

An important feature of gradient descent, in any metric, is that for $\eta$ small enough, the step is guaranteed to decrease the value of $f$.

The choice of a metric $A$ represents the choice of a norm for vectors in parameter space. Conversely, choosing a set of parameters and using the "naive" gradient ascent for these parameters amounts to implicitly deciding that these parameters form an orthonormal basis.

For the neural network above, the gradient ascent $w_{i j} \leftarrow w_{i j}-\eta \frac{\partial \ell}{\partial w_{i j}}$ corresponds to the choice of $A=\mathrm{Id}$ on parameter space, namely, the norm of a change of parameters $\delta w=\left(\delta w_{i j}\right)$ is $\|\delta w\|^{2}:=\sum\left|\delta w_{i j}\right|^{2}$. Thus, gradient descent for $w_{i j}$ gives the best $\delta w$ for a given norm $\|\delta w\|$, that is, the best change of $f$ for a given change in the numerical value of the parameters.

Example: from sigmoid to tanh activation function. Neural networks using the sigmoid and tanh activation function are defined, respectively, by

$$
a_{k}=\operatorname{sigm}\left(\sum_{i, i \rightarrow k} a_{i} w_{i k}\right)
$$

and

$$
a_{k}^{\prime}=\tanh \left(\sum_{i, i \rightarrow k} a_{i}^{\prime} w_{i k}^{\prime}\right)
$$

(including the biases $w_{0 k}$ and $\left.w_{0 k}^{\prime}\right)$. Since $\tanh (x)=2 \operatorname{sigm}(2 x)-1$, the activities of the network correspond to each other via $a_{k}^{\prime}=2 a_{k}-1$ for all $k$ if we set

$$
w_{i k}^{\prime}=\frac{w_{i k}}{4}
$$

for $i \neq 0$, and

$$
w_{0 k}^{\prime}=\frac{w_{0 k}}{2}+\frac{1}{4} \sum_{i \neq 0} w_{i k}
$$


for the biases.

Consequently, while the gradient for the sigmoid function will try to improve performance while minimizing the change to the numerical values of $w_{i k}$ and $w_{0 k}$, the gradient for the tanh function will do the same for the numerical values of $w_{i k}^{\prime}$ and $w_{0 k}^{\prime}$, obviously resulting in different updates. If we follow the tanh gradient and rewrite it back in terms of the variables $w_{i k}$, we see that the tanh update expressed in the variables $w_{i k}$ is

$$
w_{i k} \leftarrow w_{i k}+16\left(\delta w_{i k}-\frac{1}{2} \delta w_{0 k}\right) \quad(i \neq 0)
$$

and

$$
w_{0 k} \leftarrow w_{0 k}+4 \delta w_{0 k}-8 \sum_{i \neq 0}\left(\delta w_{i k}-\frac{1}{2} \delta w_{0 k}\right)
$$

where $\delta w_{i k}$ is the update that would have been applied to $w_{i k}$ if we were following the standard sigmoid backpropagation. Indeed this takes the form of a symmetric matrix applied to $\delta w_{i k}$ (the cross-contributions of $\delta w_{0 k}$ to $w_{i k}$ and of $\delta w_{i k}$ to $w_{0 k}$ are the same).

Apart from an obvious speedup factor, an important difference between this update and ordinary (sigmoid) backpropagation on the $w_{i k}$ is that each time a weight $w_{i k}$ is updated, there is an opposite, twice as small contribution to $w_{0 k}$ : in this sense, it is as if this update assumes that the activities $a_{i}$ are centered around $1 / 2$ so that when $w_{i k}$ gets changed to $w_{i k}+c$, one "needs" to add $-c / 2$ to the bias so that things stay the same on average.

Newton's method and gradient descent. To find the minimum of a function $f$ on $\mathbb{R}$, one can use the Newton method to solve $f^{\prime}=0$, namely, $x \leftarrow x-f^{\prime}(x) / f^{\prime \prime}(x)$. In higher dimension this becomes

$$
x \leftarrow x-(\operatorname{Hess} f)^{-1} \partial f / \partial x
$$

where $\partial f / \partial x$ is the vector of partial derivatives, and (Hess $f)_{i j}:=\partial^{2} f / \partial x_{i} \partial x_{j}$ is the Hessian matrix of $f$.

Around a non-degenerate minimum of $f$, the Hessian Hess $f$ will be a positive-definite matrix. So the Newton method can be seen as a gradient descent with learning rate $\eta=1$, in the metric $A=\operatorname{Hess} f$, when one is close enough to a minimum.

Intrinsic metrics. There could be a lot of arguing and counter-arguing about the "right" way to write the parameters with respect to which the gradient should be taken. The solution to avoid these choices is known: use metrics that depend on what the system does, rather than on how the parameters are decomposed as numbers. 
The Fisher metric, which defines a natural gradient [AN00, is one such metric. Namely: the size (norm) of a change of parameters is measured by the change it induces on the probability distribution of the output of the model. The symmetric matrix $A$ used in the gradient update is then the Fisher information matrix. We will use scaled-down versions of the Fisher metric for better scalability.

We present another metric for neural networks, the backpropagated metric. The size of a change of parameters at a given unit is measured by the effect it has on the units it directly influences, which is itself measured recursively in the same way up to the output layer. The matrix defining this metric is obtained by well-known equations related to the Gauss-Newton approximation of the Hessian.

\subsection{Intrinsic metrics and their computation by backpropaga- tion}

Here we rewrite the definition of neural networks in the language of differential manifolds and Riemannian geometry; this allows us to define metrics directly in an intrinsic way.

Consider a neural-like network made of units influencing each other. The activity of each unit $k$ takes values in a space $\mathcal{A}_{k}$ which we assume to be a differentiable manifold (typically $\mathbb{R}$ without a preferred origin and scale, but we allow room for multidimensional activations). Suppose that the activation of the network follows

$$
a_{k}=f_{\theta_{k}}^{k}\left(a_{i_{1}}, \ldots, a_{i_{n_{k}}}\right)
$$

where $a_{i_{1}}, \ldots, a_{i_{n_{k}}}$ are the units pointing to $k$, and where $f_{\theta_{k}}^{k}$ is a function from $\mathcal{A}_{i_{1}} \times \cdots \times \mathcal{A}_{i_{n_{k}}}$ to $\mathcal{A}_{k}$, depending on a parameter $\theta_{k}$ which itself belongs to a manifold $\Theta_{k}$. Here we have no special, always-activated unit coding for biases: the biases are a part of the parameters $\theta_{k}$.

We shall also assume that the output units in the network are interpreted through a final decoding function to produce an object $\omega=\omega\left(\left(a_{k}\right)_{k \in \mathcal{L}_{\text {out }}}\right)$ relevant to the initial problem, also assumed to belong to a differentiable manifold.

To implement any gradient ascent over the parameters $\theta$, we first need a (Riemannian) metric on the parameter space. Such a metric can be defined by choosing a parametrization by $\mathbb{R}^{d}$ and deciding that the elementary vectors of $\mathbb{R}^{d}$ are orthogonal, but this is not intrinsic: different parametrizations will lead to different learning trajectories.

In this setting, an object is said to be intrinsic if it does not depend on a choice of parametrization of any of the manifolds involved (activities, parameters, final output). Hopefully, casting the activities as elements in an abstract manifold, and writing intrinsic algorithms that will not depend 
on how this manifold is represented as numbers, allows the algorithms to be agnostic as to any physical interpretation of these activities (activation levels, activation frequencies, log-frequencies, synchronized activity of a group of neurons...)

We assume that we are given a meaningful Riemannian metric on the final output $\omega$ : that is, we know how to measure the size of a change in the output. For instance, if $\omega$ describes a probability distribution over a target variable $y$, we can use the Fisher metric over $\omega$ (usually simpler to work with than the Fisher metric over the whole network parameter space). In the case $\omega$ is a Gaussian of fixed variance centered on the output values, this coincides with a Euclidean norm on these values.

Then there are several possibilities to define intrinsic Riemannian metrics on parameter space. The most direct one is the Fisher metric: the output is seen as a function of all parameters, and the norm of a change of parameter $\delta \theta$ (over all parameters at once) is the norm of the change it induces on the output. This is not scalable: for a neural network with $n$ units, $n_{\text {out }}$ output units, and $d$ incoming edges per unit, processing each data sample takes $O\left(n^{2} d^{2}+n^{2} n_{\text {out }}\right)$, compared to $O(n d)$ for backpropagation.

A more scalable version is to break down the change of parameter into a sum of changes of incoming parameters to each unit and take the Fisher metric at each unit independently. This is the unitwise Fisher metric. As we will see, it scales well to sparsely connected networks if the output layer is not too large: processing each data sample takes $O\left(n d^{2}+n_{\text {out }} n d\right)$.

An even simpler version is the backpropagated metric, defined by backwards induction from the output layer: the norm of a change of parameter on the output layer is the norm of the change it induces on the final result, and the norm of a change of parameter at an internal unit is the sum of the norm of the resulting changes at the units it influences directly. Processing each data sample takes $O\left(n d^{2}\right)$.

Quasi-diagonal reduction (Section 2.3) further produces simplified intrinsic metrics in which the $O\left(n d^{2}\right)$ terms reduce to $O(n d)$.

Notation. In what follows, we use the standard objects of differential geometry but try to present them in an intuitive way; Appendix C gives a fully formal treatment. The notation $\delta a, \delta \theta, \delta \omega$ denotes tangent vectors on the corresponding manifolds (intuitively, differences between two very close values of $a$ or $\theta$ or $\omega$ ). The notation $\frac{\partial a_{i}}{\partial a_{k}}$ denotes the differential (total derivative) of the activity $a_{i}$ seen as a function of $a_{k}$. In a basis it is represented as the Jacobian matrix of partial derivatives of the components of $a_{i}$ w.r.t. those of $a_{k}$ (if activities are more than 1-dimensional). In particular, for a numerical function $f, \frac{\partial f}{\partial \theta}$ is represented as a row vector, and for an infinitesimal change (tangent vector) $\delta \theta$ we have $\delta f=\frac{\partial f}{\partial \theta} \delta \theta$. The various metrics involved are $(0,2)$-tensors, but we use standard matrix notation for them. With this convention a metric gradient descent on $\theta$ takes the form 
$\theta \leftarrow \theta-M(\theta)^{-1} \frac{\partial f^{\top}}{\partial \theta}$.

DEFinition 13 (NATURAL METRIC, UNITWISE NATURAL METRIC, BACKPROPAGATED METRIC). Let $\|\delta \omega\|^{2}=\sum \Omega_{i j} \delta \omega_{i} \delta \omega_{j}=\delta \omega^{\top} \Omega \delta \omega$ be a metric on the final output of the network, given by the symmetric, positivedefinite matrix $\Omega$. We define three metrics on the parameter set.

- The natural metric on the parameter set $\theta=\left(\theta_{k}\right)$ is defined as follows. Let $x$ be an input in the dataset $\mathcal{D}$ and let $\omega(x)$ be the final output of the network run with input $x$ and parameter $\theta$. Let $\delta \theta$ be a variation of $\theta$ and let $\delta \omega(x)$ be the resulting variation of $\omega(x)$. Let

$$
\|\delta \theta\|_{\text {nat }, x}^{2}:=\|\delta \omega(x)\|^{2}
$$

and then define the natural metric by

$$
\|\delta \theta\|_{\text {nat }}^{2}:=\mathbb{E}_{x \in \mathcal{D}}\|\delta \theta\|_{\text {nat }, x}^{2}
$$

In matrix form, we have $\delta \omega(x)=\frac{\partial \omega(x)}{\partial \theta} \delta \theta$ where $\frac{\partial \omega}{\partial \theta}$ is the Jacobian matrix of $\omega(x)$ as a function of $\theta$, so that the natural metric is given by the matrix

$$
\|\delta \theta\|_{\text {nat }}^{2}=\mathbb{E}_{x \sim \mathcal{D}} \delta \theta^{\top} \frac{\partial \omega(x)^{\top}}{\partial \theta} \Omega \frac{\partial \omega(x)}{\partial \theta} \delta \theta
$$

The natural metric is given by a matrix of $\operatorname{size} \operatorname{dim} \theta=\sum_{k} \operatorname{dim} \theta_{k}$.

- The unitwise natural metric on the parameter set $\theta$ is

$$
\|\delta \theta\|_{\mathrm{u}-\mathrm{nat}}^{2}:=\sum_{k}\left\|\delta \theta_{k}\right\|_{\text {nat }}^{2}
$$

where $k$ runs over the units of the network and $\delta \theta_{k}$ is the variation of the incoming parameters to unit $k$. This metric is given by keeping only the block-diagonal terms incoming to each unit in the matrix defining the natural metric.

In case $\omega$ is a probability distribution and the metric $\Omega$ on $\omega$ is the Fisher metric, we also call $\|\delta \theta\|_{\text {nat }}$ and $\|\delta \theta\|_{\mathrm{u}-\text { nat }}$ the Fisher metric and unitwise Fisher metric.

- The backpropagated metric on $\theta$ is defined as follows. Let $x$ be an input in the data. We first define a metric on each of the activities $a_{k}$, depending on the input $x$, working from the output layer backwards.

Given a change $\delta a_{k_{\text {out }}}$ in the activity at an output unit $k_{\text {out }}$, let $\delta \omega(x)$ be the corresponding change in the final output and set

$$
\left\|\delta a_{k_{\mathrm{out}}}\right\|_{\mathrm{bp}, x}^{2}:=\|\delta \omega(x)\|^{2}
$$


The metric on internal units $k$ is defined as follows: Given a change $\delta a_{k}$ in the activity of unit $k$, let $\delta a_{i}$ be the resulting changes in the activities of units $k \rightarrow i$ directly influenced by $k$. Define by induction from the output layer

$$
\left\|\delta a_{k}\right\|_{\mathrm{bp}, x}^{2}:=\sum_{i, k \rightarrow i}\left\|\delta a_{i}\right\|_{\mathrm{bp}, x}^{2}
$$

Given a change $\delta \theta_{k}$ of the incoming parameters to unit $k$, let $\delta a_{k, x}$ be the change of activity of unit $k$ resulting from the change $\delta \theta_{k}$, when the network is run on input $x$. Define the backpropagated metric by

$$
\left\|\delta \theta_{k}\right\|_{\mathrm{bp}}^{2}:=\mathbb{E}_{x \in \mathcal{D}}\left\|\delta a_{k, x}\right\|_{\mathrm{bp}, x}^{2}
$$

and

$$
\|\delta \theta\|_{\mathrm{bp}}^{2}:=\sum_{k \in \mathcal{L}}\left\|\delta \theta_{k}\right\|_{\mathrm{bp}}^{2}
$$

Another metric, the outer product (OP) metric, can be defined from slightly different ingredients. It corresponds to an often-used variant of the natural gradient (e.g., APF00, RMB07]), in which the expectation under the current probability distribution is replaced with a similar term involving only the desired target $y$ for each input $x$ (more details in Section 2.5). It can readily be computed by backpropagation from (62).

Whereas the metrics above depend on the actual output $\omega(x)$ for each input $x$, together with a metric on $\omega$, but not on any target value for $x$, the OP metric depends on a loss function $\ell(\omega(x), y(x))$ encoding the deviation of $\omega(x)$ from a desired target $y(x)$ for $x$; but not on a choice of metric for $\omega$.

Definition 14 (Outer product metric). For each input $x$ in the dataset $\mathcal{D}$, let $\omega(x)$ be the final output of the network run with input $x$ and parameter $\theta$. Let $\ell(\omega(x), y(x))$ be the loss function measuring how $\omega(x)$ departs from the desired output $y(x)$ for $x$.

The outer product metric is defined as follows. Let $\delta \theta$ be a variation of $\theta$ and let $\delta \ell_{x}$ be the resulting variation of $\ell(\omega(x), y(x))$. Define

$$
\|\delta \theta\|_{\text {op }}^{2}:=\mathbb{E}_{x \in \mathcal{D}}\left(\delta \ell_{x}\right)^{2}
$$

In matrix form, this metric is

$$
\|\delta \theta\|_{\mathrm{op}}^{2}=\mathbb{E}_{x \sim \mathcal{D}} \delta \theta^{\top} \frac{\partial \ell}{\partial \theta} \frac{\partial \ell}{\partial \theta} \delta \theta
$$

where $\frac{\partial \ell}{\partial \theta}$ is the row vector of partial derivatives (the differential) of the loss function. Thus this metric is given by the matrix

$$
\mathbb{E}_{x \sim \mathcal{D}} \frac{\partial \ell^{\top}}{\partial \theta} \frac{\partial \ell}{\partial \theta}
$$


hence its name.

The unitwise outer product metric is defined by

$$
\|\delta \theta\|_{\mathrm{u}-\mathrm{op}}^{2}:=\sum_{k}\left\|\delta \theta_{k}\right\|_{\mathrm{op}}^{2}
$$

where $k$ runs over the units of the network and $\delta \theta_{k}$ is the variation of the incoming parameters to unit $k$. This metric is given by keeping only the block-diagonal terms incoming to each unit in the matrix defining the tensorsquare differential metric.

The OP metric has been used simply under the name "natural gradient" in APF00, RMB07, which can lead to some confusion because it is distinct from the natural metric using the true Fisher information matrix (see the discussion in [PB13]). Moreover the OP metric makes sense for optimization situations more general than the natural gradient, in which the loss function is not necessarily of the form $\ln p_{\theta}$ for a probabilistic model $p$. For these two reasons we adopt a purely descriptive nam 8 .

The OP metric is characterized, among all possible metrics, by a unique property: it provides a gradient step for which progress is most evenly distributed among all data samples.

Proposition 15 (OP GRAdient equalizes the Gain over the SAMPLES). Let $L:=\mathbb{E}_{x \in \mathcal{D}} \ell_{x}$ be the average loss with $\ell_{x}$ the loss on input $x$. The direction $\delta \theta$ given by the gradient of $L$ computed in the outer product metric (Def. 26) has the following property: Among all directions $\delta \theta$ yielding the same infinitesimal increment $\delta L$ at first order, it is the one for which the increment is most evenly spread over the data samples $x \in \mathcal{D}$, namely, $\operatorname{Var}_{x \in \mathcal{D}} \delta \ell_{x}=\mathbb{E}_{x \in \mathcal{D}}\left(\delta \ell_{x}-\delta L\right)^{2}$ is minimal.

The proof is given in the Appendix. The unitwise OP metric does not, in general, satisfy this property: instead, it minimizes the variance, over a random data sample $x \in \mathcal{D}$ and a random unit $k$ in the network, of the contribution to $\delta \ell_{x}$ of the change $\delta \theta_{k}$ at unit $k$, so that it tries to spread $\delta L$ uniformly both over data samples and units.

REMARK 16 (Metric For outPut PARAmeters). The case when the decoding function $\omega\left(\left(a_{k}\right)_{k \in \mathcal{L}_{\text {out }}}\right)$ depends on additional "output parameters" $\vartheta$ (e.g., softmax output with variable coefficients $\operatorname{Pr}(k)=\mathrm{e}^{\vartheta_{k} a_{k}} /\left(\sum_{k^{\prime}} \mathrm{e}^{\left.\left.\vartheta_{k^{\prime}} a_{k^{\prime}}\right)\right)}\right.$ can be recovered by considering $\omega$ as an additional output unit to the network, so that $\vartheta$ becomes the parameter of the activation function of $\omega$. In

\footnotetext{
8 The outer product metric is distinct from the "outer product (or LevenbergMarquardt) approximation" of the Hessian of the loss function [Bis06, 5.4.2]. The latter can be obtained from the natural metric (54) in which the output metric $\Omega$ is replaced with the Hessian of the loss function $\ell_{x}$ w.r.t. $\omega$. It depends on the parametrization of $\omega$. For exponential families in the canonical parametrization it coincides with the Fisher metric.
} 
particular, applying the definitions above, the metric $\Omega$ on $\omega$ induces a metric on $\vartheta$ by

$$
\|\delta \vartheta\|_{\mathrm{bp}}^{2}=\|\delta \vartheta\|_{\text {nat }}^{2}=\delta \vartheta^{\top}\left(\mathbb{E}_{x \in \mathcal{D}} \frac{\partial \omega^{\top}}{\partial \vartheta} \Omega \frac{\partial \omega}{\partial \vartheta}\right) \delta \vartheta
$$

given by the matrix $\mathbb{E}_{x \in \mathcal{D}} \frac{\partial \omega}{\partial \vartheta} \Omega \frac{\partial \omega}{\partial \vartheta}$. So $\vartheta$ can be trained by gradient descent in this metric.

For the Fisher metric, the following is well-known.

Proposition 17 (Invariance). The natural metric, unitwise natural metric, backpropagated metric, and plain and unitwise outer product metrics are intrinsic: $\|\delta \theta\|_{\text {nat }},\|\delta \theta\|_{\mathrm{u}-\mathrm{nat}},\|\delta \theta\|_{\mathrm{bp}},\|\delta \theta\|_{\mathrm{op}}$, and $\|\delta \theta\|_{\mathrm{u}-\mathrm{op}}$ do not depend on a choice of parametrization for the activations $a_{k}$ and for the parameter $\theta_{k}$ at each unit $k$.

Proof. These metrics have been defined without any reference to parametrizations, directly by defining the norm of a tangent vector $\delta \theta$; see Appendix C for a more formal treatment. Consequently the value of the norm $\|\delta \theta\|$ is the same expressed in any coordinate system [GHL87, 2.1].

The natural metric actually has stronger invariance properties than the unitwise natural metric: it does not depend on a change of parametrization of the whole parameter $\theta=\left(\theta_{k}\right)$ that would mix the various components. As such, the unitwise natural metric depends on a choice of decomposition of the network into units, while the natural metric is only a function of the input-output relationship of the whole network. The same holds for the OP and unitwise OP metrics.

REMARK 18 (UNITWISE METRICS AS CHANGE IN ACTIVATION PROFILE). We saw above that the metric used to define a gradient represents a "cost" of moving in certain directions. All three unitwise metrics (unitwise natural, backpropagated, and unitwise OP) share a common property: these metrics decompose as a sum, over the units $k$, of terms of the form $\left\|\delta \theta_{k}\right\|^{2}=\mathbb{E}_{x \in \mathcal{D}} c_{x, k}\left\|\delta a_{k}(x)\right\|^{2}$ where $\delta a_{k}(x)$ is the resulting change of activity at $k$ on input $x$, and $c_{x, k}$ is a weight (different for these three metrics) estimating the influence of $k$ on the output. Thus, the "cost" of a change at unit $k$ according to these metrics, is an average square norm of the resulting change in activation profile $a_{k}(x)$ over $x$ in the dataset. This is related to the best-fit interpretation of these metrics (Section 3.3). 
Computing the metrics. These metrics can be explicitly computed as follows.

The outer product metric is the easiest to compute: the terms $\frac{\partial \ell}{\partial \theta}$ are directly computed by ordinary backpropagation, namely, $\frac{\partial \ell}{\partial \theta_{k}}=\frac{\partial \ell}{\partial a_{k}} \frac{\partial a_{k}}{\partial \theta_{k}}$ where $\frac{\partial \ell}{\partial a_{k}}\left(=b_{k}\right)$ is computed by backpropagation and $\frac{\partial a_{k}}{\partial \theta_{k}}$ is obtained from the activation function at unit $k$. Then the matrix defining the metric is $\mathbb{E}_{x \in \mathcal{D}} \frac{\partial \ell^{\top}}{\partial \theta} \frac{\partial \ell}{\partial \theta}$, or, for the unitwise version, $\mathbb{E}_{x \in \mathcal{D}} \frac{\partial \ell}{\partial \theta_{k}} \frac{\partial \ell}{\partial \theta_{k}}$ at each unit $k$.

To compute the natural and unitwise natural metrics, it is enough to compute the Jacobian matrix $\frac{\partial \omega}{\partial \theta}$. This can be done by performing one backpropagation for each component of the output layer, for each input $x \in \mathcal{D}$, as follows.

Definition 19 (BACKPropagation transfer rates). Let $k_{\text {out }}$ be an output unit and let $k$ be any unit in the network. The backpropagation transfer rate $J_{k}^{k_{\text {out }}}$ from $k_{\text {out }}$ to $k$ is the $\operatorname{dim}\left(a_{k_{\text {out }}}\right) \times \operatorname{dim}\left(a_{k}\right)$ matrix defined by

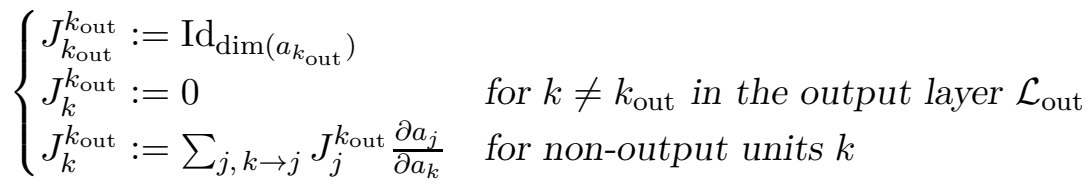

where $\frac{\partial a_{j}}{\partial a_{k}}$ is the Jacobian matrix of the activation function from unit $k$ to unit $j$. Then we have $J_{k}^{k_{\text {out }}}=\frac{\partial a_{k_{\text {out }}}}{\partial a_{k}}$.

This depends on an input $x$ : the activation state of the network has to be computed by forward propagation before these quantities can be computed.

Typically the activities are one-dimensional, not multidimensional, so that each $J_{k}^{k_{\text {out }}}$ is just a number, not a matrix. In this case, all the transfer rates $J_{k}^{k_{\text {out }}}$ can be computed by performing $n_{\text {out }}$ distinct backpropagations each initialized with a single 1 on the output layer.

Since the influence of the parameter $\theta_{k}$ on the output goes through the activity of unit $k$, the unitwise natural metric at $k$ can be computed from a single number (if activities are one-dimensional) measuring the influence of unit $k$ on the output, the Fisher modulus.

Definition 20 (Fisher modulus). Let $x$ be an input. Let $k$ be a unit in the network. Let $\Omega$ be the metric on the final output $\omega$. The Fisher modulus $\Phi_{k}(x)$ of $k$ on input $x$ is the $\operatorname{dim}\left(a_{k}\right) \times \operatorname{dim}\left(a_{k}\right)$ matrix given by

$$
\Phi_{k}(x):=\left(\sum_{k_{\text {out }} \in \mathcal{L}_{\text {out }}} \frac{\partial \omega}{\partial a_{k_{\text {out }}}} J_{k}^{k_{\text {out }}}\right)^{\top} \Omega\left(\sum_{k_{\text {out }} \in \mathcal{L}_{\text {out }}} \frac{\partial \omega}{\partial a_{k_{\text {out }}}} J_{k}^{k_{\text {out }}}\right)
$$

For each input $x$, the Fisher modulus is an intrinsic metric on $a_{k}$ : for a given input $x$, the norm

$$
\left\|\delta a_{k}\right\|_{\mathrm{F}-\bmod }^{2}:=\delta a_{k}^{\top} \Phi_{k} \delta a_{k}
$$


does not depend on any choice of parametrization.

Note that $\frac{\partial \omega}{\partial a_{k_{\text {out }}}}$ depends on the output layer interpretation but not on any parameter $\theta$. Thus, since the transfer rates $J$ can be computed by backpropagation, the Fisher modulus only involves known quantities.

Proposition 21 (Computation of the Unitwise natural metRIC). The unitwise natural metric at unit $k$ is given by

$$
\begin{aligned}
\left\|\delta \theta_{k}\right\|_{\mathrm{u}-\mathrm{nat}}^{2} & =\mathbb{E}_{x \in \mathcal{D}}\left\|\delta a_{k}(x)\right\|_{\mathrm{F}-\bmod }^{2} \\
& =\mathbb{E}_{x \in \mathcal{D}} \delta \theta_{k}^{\top} \frac{\partial a_{k}^{\top}}{\partial \theta_{k}} \Phi_{k} \frac{\partial a_{k}}{\partial \theta_{k}} \delta \theta_{k}
\end{aligned}
$$

where $\delta a_{k}(x)$ is the variation of $a_{k}(x)$ induced by $\delta \theta$, and $\frac{\partial a_{k}}{\partial \theta_{k}}$ is the Jacobian matrix of the activation function at $k$. Thus the matrix defining the unitwise natural metric at unit $k$ is

$$
F^{(k)}=\mathbb{E}_{x \in \mathcal{D}} \frac{\partial a_{k}^{\top}}{\partial \theta_{k}} \Phi_{k} \frac{\partial a_{k}}{\partial \theta_{k}}
$$

Proof. By definition of the transfer rates $J$ we have $J_{k}^{k_{\text {out }}}=\frac{\partial a_{k_{\text {out }}}}{\partial a_{k}}$. Thus $\sum_{k_{\text {out }} \in \mathcal{L}_{\text {out }}} \frac{\partial \omega}{\partial a_{k_{\text {out }}}} J_{k}^{k_{\text {out }}}=\frac{\partial \omega}{\partial a_{k}}$ so that

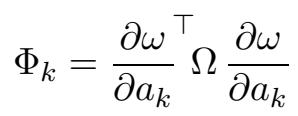

hence

$$
\frac{\partial a_{k}^{\top}}{\partial \theta_{k}} \Phi_{k} \frac{\partial a_{k}}{\partial \theta_{k}}=\frac{\partial a_{k}{ }^{\top}}{\partial \theta_{k}} \frac{\partial \omega^{\top}}{\partial a_{k}} \Omega \frac{\partial \omega}{\partial a_{k}} \frac{\partial a_{k}}{\partial \theta_{k}}=\frac{\partial \omega^{\top}}{\partial \theta_{k}} \Omega \frac{\partial \omega}{\partial \theta_{k}}
$$

which, after averaging over the dataset, is the definition of the unitwise natural metric at $k$.

An analogous formula can be defined for the full (rather than unitwise) Fisher matrix, by defining a Fisher modulus $\Phi_{k k^{\prime}}$ indexed by two units, and using unit $k^{\prime}$ on the left and $k$ on the right in (66). Then the block of entries of the Fisher matrix corresponding to parameters $\theta_{k}$ and $\theta_{k^{\prime}}$ is

$$
\mathbb{E}_{x \in \mathcal{D}} \frac{\partial a_{k^{\prime}}{ }^{\top}}{\partial \theta_{k^{\prime}}} \Phi_{k k^{\prime}} \frac{\partial a_{k}}{\partial \theta_{k}}
$$

(see also Proposition [27).

The unitwise Fisher metric is costly to compute when the output layer is large. We can define another intrinsic metric for the activation of unit $k$, simply by backpropagating the metric of the output layer. 
The changes in the output induced by a change of $\theta_{k}$ all transit through the activation of unit $k$. So if we have an intrinsic metric $\left\|\delta a_{k}\right\|^{2}$ for the activation of unit $k$, we can immediately define an intrinsic metric for $\theta_{k}$, by looking at the resulting change $\delta a_{k}=\frac{\partial a_{k}}{\partial \theta_{k}} \delta \theta_{k}$ induced by a change $\delta \theta_{k}$, and defining the norm of $\delta \theta_{k}$ to be the norm of the resulting $\delta a_{k}$. If the metric on $a_{k}$ is given, in some parametrization of $a_{k}$, by $\left\|\delta a_{k}\right\|^{2}=\delta a_{k}^{\top} g_{k} \delta a_{k}$ where $g_{k}$ is a symmetric, positive-definite matrix of size $\left(\operatorname{dim} a_{k}\right) \times\left(\operatorname{dim} a_{k}\right)$, then defining $\left\|\delta \theta_{k}\right\|$ to be the norm of this $\delta a_{k}$,

$$
\left\|\delta \theta_{k}\right\|:=\left\|\frac{\partial a_{k}}{\partial \theta_{k}} \delta \theta_{k}\right\|
$$

yields

$$
\left\|\delta \theta_{k}\right\|^{2}=\left(\frac{\partial a_{k}}{\partial \theta_{k}} \delta \theta_{k}\right)^{\top} g_{k}\left(\frac{\partial a_{k}}{\partial \theta_{k}} \delta \theta_{k}\right)
$$

in other words, the matrix defining this metric is $\frac{\partial a_{k}}{\partial \theta_{k}} g_{k} \frac{\partial a_{k}}{\partial \theta_{k}}$.

The unitwise Fisher metric is obtained from the Fisher modulus by this construction. We now define another intrinsic modulus playing the same role for the backpropagated metric.

Proposition 22 (BACKPropagated modulus and COMPUtaTION OF THE BACKPROPAGATED METRIC). Let $x$ be an input. Let $k$ be a unit in the network. Let $\Omega$ be the metric on the final output $\omega$. The backpropagated modulus $m_{k}(x)$ at $k$ is the $\operatorname{dim}\left(a_{k}\right) \times \operatorname{dim}\left(a_{k}\right)$ matrix given by

$$
m_{k}(x):= \begin{cases}\frac{\partial \omega}{\partial a_{k}} \Omega \frac{\partial \omega}{\partial a_{k}} & \text { for } k \text { in the output layer } \\ \sum_{j, k \rightarrow j} \frac{\partial a_{j}}{\partial a_{k}} m_{j} \frac{\partial a_{j}}{\partial a_{k}} & \text { for } k \text { an internal unit }\end{cases}
$$

Then, for each input $x$, the backpropagated metric on $a_{k}$ is given by the backpropagated modulus:

$$
\left\|\delta a_{k}\right\|_{\mathrm{bp}, x}^{2}=\delta a_{k}^{\top} m_{k} \delta a_{k}
$$

and so the backpropagated metric on $\theta_{k}$ is given by the matrix $\mathbb{E}_{x \in \mathcal{D}} \frac{\partial a_{k}}{\partial \theta_{k}} m_{k} \frac{\partial a_{k}}{\partial \theta_{k}}$, namely,

$$
\left\|\delta \theta_{k}\right\|_{\mathrm{bp}}^{2}=\mathbb{E}_{x \in \mathcal{D}} \delta \theta_{k}^{\top} \frac{\partial a_{k}^{\top}}{\partial \theta_{k}} m_{k} \frac{\partial a_{k}}{\partial \theta_{k}} \delta \theta_{k}
$$

Proof. Immediate from the definition of the backpropagated metric and $\delta a_{i}=\frac{\partial a_{i}}{\partial a_{k}} \delta a_{k}$ and $\delta a_{k}=\frac{\partial a_{k}}{\partial \theta_{k}} \delta \theta_{k}$.

Like the Fisher modulus, the backpropagated modulus is a single number when activities are one-dimensional. The cost of its computation is the same as one backpropagation pass.

The equation defining the backpropagated modulus is well-known: it is related to the so-called Gauss-Newton approximation to the Newton method 
(see for instance [LBOM96], Section 7), which consists in computing the Hessian of the loss function and throwing away all terms involving the second derivative of the activation function (those could result in non-positivedefinite terms, in which case the Newton method is ill-behaved), with the additional approximation that cross-terms between different units are also thrown away. Here we see that no approximation is involved: we do not throw away annoying terms, we simply define an intrinsic metric. There is actually no meaningful notion of the Hessian of a function on manifolds [GHL87, paragraph 3.37] unless additional structure (affine, Riemannian) is given or we are at a critical point of the function; the annoying terms above are precisely the terms that prevent such a notion to exist. So one could even say, in the context of differential geometry, that the Newton method is an approximation of the backpropagated metric rather than the other way round.

The backpropagated modulus and the Fisher modulus are related: If one tries to write a backpropagated equation to compute the Fisher modulus $\Phi_{k}$ in terms of the Fisher modulus at units pointed by $k$, one finds a quadratic (instead of linear) backpropagation equation with terms involving pairs of units. Keeping only the terms involving a single unit yields the equation defining the backpropagated modulus.

\subsection{Quasi-diagonal reduction of a unitwise metric}

The unitwise Fisher metric, backpropagated metric, and unitwise OP metric, still involve a full matrix on the incoming parameter space at each unit, and are thus not adapted if network connectivity is large. We now introduce two metrics enjoying lesser invariance properties than the above, but quicker to compute.

Given an intrinsic metric $\left\|\delta \theta_{k}\right\|$ on $\theta_{k}$ (such as the unitwise Fisher or backpropagated metric), we are going to define a simpler one, $\left\|\delta \theta_{k}\right\|_{\mathrm{qd}}$. The inverse of the matrix defining this metric will be quasi-diagonal, with the only non-zero diagonal terms being those between a weight and the bias. This will allow for quick gradient steps costing no more than classical backpropagation.

This relies on the affine structure in neural networks: this simplification makes sense in a somewhat more restricted setting than the general setting above. Suppose that the activation function

$$
a_{k}=f_{\theta_{k}}^{k}\left(a_{i_{1}}, \ldots, a_{i_{n_{k}}}\right)
$$

can be written as a composition of a fixed, non-linear activation function $\varphi$ and a quantity $y_{k}$ that is an affine function of $a_{i_{1}}, \ldots, a_{i_{n_{k}}}$ :

$$
a_{k}=\varphi\left(y_{k, \theta_{k}}\left(a_{i_{1}}, \ldots, a_{i_{n_{k}}}\right)\right)
$$


such that when $\theta_{k}$ ranges over its values, $y_{k, \theta_{k}}$ ranges over all possible affine functions of $a_{i_{1}}, \ldots, a_{i_{n_{k}}}$. For this to make sense, we now have to assume that the activities $a_{k}$ live in an affine space. So let us go back to activities with values in $\mathbb{R}$, but without any preferred origin and scale for activities: we look for invariance under replacement of $a_{i}$ with $\alpha_{i} a_{i}+\beta_{i}$ and likewise for $y_{i}$.

In any given parametrization (choice of origin and basis) of $a_{i}$ and $y_{k}$ we can write

$$
y_{k}=\sum_{i, i \rightarrow k} w_{i k} a_{i}+w_{0 k}
$$

for some values $w_{i k}$; specifying the parameter $\theta_{k}$ is equivalent to specifying these quantities.

But this decomposition will change if we change the affine parametrization of activities: if $a_{i}^{\prime}=\alpha_{i} a_{i}+\beta_{i}$ and $y_{k}^{\prime}=\gamma_{k} y_{k}+\delta_{k}$ the relation becomes $y_{k}^{\prime}=\delta_{k}+\sum_{i} \gamma_{k} w_{i k} \alpha_{i}^{-1}\left(a_{i}^{\prime}-\beta_{i}\right)+\gamma_{k} w_{0 k}=\sum_{i}\left(\gamma_{k} w_{i k} \alpha_{i}^{-1}\right) a_{i}^{\prime}+\left(\gamma_{k} w_{0 k}+\delta_{k}-\right.$ $\left.\sum_{i} w_{i k} \alpha_{i}^{-1} \beta_{i}\right)$ so that the new weights are $w_{i k}^{\prime}=\gamma_{k} w_{i k} \alpha_{i}^{-1}$ and the new bias is $w_{0 k}^{\prime}=\gamma_{k} w_{0 k}+\delta_{k}-\sum_{i} w_{i k} \alpha_{i}^{-1} \beta_{i}$. In particular we see that there is no intrinsic "separation" between the bias and the weights; but that there is a separation between $w_{i k}$ and $w_{i^{\prime} k}$ for different incoming units $i$ and $i^{\prime}$. This is formalized as follows.

Let $\delta \theta_{k}$ be a change of parameter $\theta_{k}$. For $i \neq 0$, let $\delta w_{i k}$ be the resulting change of $w_{i k}$ in a given parametrization, and let $\delta w_{i k}^{\prime}$ be the resulting change in another parametrization. If $\delta w_{i k}=0$, then we have $\delta w_{i k}^{\prime}=0$ as well in any other affine parametrization, since $w_{i k}^{\prime}=\gamma_{k} w_{i k} \alpha_{i}^{-1}$ yields $\delta w_{i k}^{\prime}=\gamma_{k} \delta w_{i k} \alpha_{i}^{-1}$. Note that this does not depend on the input $x$ either 9 . Thus, having $\delta w_{i k}=0$ is a property of $\delta \theta_{k}$ that does not depend on the chosen affine parametrization of activities: it is an intrinsic property of the change of parameter $\delta \theta_{k}$. Say that a change of parameter $\delta \theta_{k}$ does not involve unit $i$ if $\delta w_{i k}$ vanishes.

For the bias the situation is different: the expression $w_{0 k}^{\prime}=\gamma_{k} w_{0 k}+\delta_{k}-$ $\sum_{i} w_{i k} \alpha_{i}^{-1} \beta_{i}$ giving the bias in a parametrization from the bias in another parametrization is more complex, and so the fact that $\delta w_{0 k}=0$ does depend on the parametrization. This is where the metric $\left\|\delta \theta_{k}\right\|$ we are trying to simplify comes into play.

Say that a change of parameter $\delta \theta_{k}$ is pure bias if it does not involve any unit $i$ incoming to $k$, i.e., if $\delta w_{i k}=0$ for all $i \neq 0$. This is an intrinsic condition. Say that $\delta \theta_{k}$ is bias-free if it is orthogonal, in the metric $\left\|\delta \theta_{k}\right\|$ we are trying to simplify, to all pure-bias vectors. Being bias-free is an intrinsic condition, because by assumption the metric $\left\|\delta \theta_{k}\right\|$ is intrinsic. Being biasfree does not simply mean $\delta w_{0 k}=0$; let us work it out in coordinates.

\footnotetext{
${ }^{9}$ This relies on the affine form (81) of $y_{k}$. If $y_{k}$ is not affine in (80), having a constant $\partial y_{k} / \partial a_{i}$ is not a well-defined notion and may depend on the input.
} 
Let $A_{i i^{\prime}}$ be the symmetric matrix defining the metric $\left\|\delta \theta_{k}\right\|$ in a given parametrization. The associated scalar product is

$$
\left\langle\delta \theta_{k}, \delta \theta_{k}^{\prime}\right\rangle=\sum_{i} \sum_{i^{\prime}} A_{i i^{\prime}} \delta w_{i k} \delta w_{i^{\prime} k}^{\prime}+\sum_{i} A_{0 i}\left(\delta w_{0 k} \delta w_{i k}^{\prime}+\delta w_{0 k}^{\prime} \delta w_{i k}\right)+A_{00} \delta w_{0 k} \delta w_{0 k}^{\prime}
$$

with $A_{0 i}=A_{i 0}$.

In particular, if the only non-zero component of $\delta \theta_{k}$ is $\delta w_{i k}$, then its scalar product with a pure bias $\delta w_{0 k}^{\prime}$ will be $A_{0 i} \delta w_{0 k}^{\prime} \delta w_{i k}$. On the other hand, if to $\delta \theta_{k}$ we add a bias component $\delta w_{0 k}=-A_{00}^{-1} A_{0 i} \delta w_{i k}$, then the scalar product with any pure bias will vanish. Such a $\delta \theta_{k}$ is thus bias-free.

In the case when the parameter $\theta_{k}$ allows to represent all affine functions of the incoming activations, we can decompose a variation $\delta \theta_{k}$ of $\theta_{k}$ into components $\delta \theta_{k i}$ each involving only one incoming unit $i$, and a pure bias component $\delta \theta_{k 0}$. This decomposition is unique if we impose that each $\delta \theta_{k i}$ is bias-free. Explicitly, if in some parametrization we have $\delta \theta_{k}=\left(\delta w_{0 k}, \delta w_{1 k}, \ldots, \delta w_{n_{k} k}\right)$ this decomposition is

$$
\delta \theta_{k i}=\left(-A_{00}^{-1} A_{0 i} \delta w_{i k}, 0, \ldots, \delta w_{i k}, 0, \ldots, 0\right)
$$

and

$$
\delta \theta_{k 0}=\left(\delta w_{0 k}+\sum_{i} A_{00}^{-1} A_{0 i} \delta w_{i k}, 0, \ldots, 0\right)
$$

The decomposition $\delta \theta_{k}=\delta \theta_{k 0}+\sum_{i} \delta \theta_{k i}$ is intrinsic.

We can then define a new intrinsic metric on $\delta \theta_{k}$ by setting

$$
\left\|\delta \theta_{k}\right\|_{\mathrm{qd}}^{2}:=\left\|\delta \theta_{k 0}\right\|^{2}+\sum_{i}\left\|\delta \theta_{k i}\right\|^{2}
$$

which is readily computed:

$$
\begin{aligned}
\left\|\delta \theta_{k}\right\|_{\mathrm{qd}}^{2}= & A_{00}\left(\delta w_{0 k}+\sum_{i} A_{00}^{-1} A_{0 i} \delta w_{i k}\right)^{2} \\
& +\sum_{i}\left(A_{i i} \delta w_{i k}^{2}-2 g_{0 i}\left(A_{00}^{-1} A_{0 i} \delta w_{i k}\right) \delta w_{i k}+A_{00}\left(A_{00}^{-1} A_{0 i} \delta w_{i k}\right)^{2}\right) \\
= & A_{00} \delta w_{0 k}^{2}+2 \sum_{i} A_{0 i} \delta w_{0 k} \delta w_{i k}+\sum_{i, i^{\prime}} A_{00}^{-1} A_{0 i} A_{0 i^{\prime}} \delta w_{i k} \delta w_{i^{\prime} k} \\
& +\sum_{i}\left(A_{i i}-A_{00}^{-1} A_{0 i}^{2}\right) \delta w_{i k}^{2}
\end{aligned}
$$

Thus, this metric is defined by a matrix $\tilde{A}$ given by $\tilde{A}_{00}=A_{00}, \tilde{A}_{0 i}=A_{0 i}$ and $\tilde{A}_{i i^{\prime}}=A_{00}^{-1} A_{0 i} A_{0 i^{\prime}}+\mathbb{1}_{i=i^{\prime}}\left(A_{i i}-A_{00}^{-1} A_{0 i}^{2}\right)$.

Definition 23. Quasi-diagonal reduction is the process which, to an intrinsic metric defined by a matrix $A$ in affine coordinates, associates the metric defined by the matrix

$$
\tilde{A}:=\operatorname{diag}(A)+A_{00}^{-1}(v \otimes v)-\operatorname{diag}\left(A_{00}^{-1}(v \otimes v)\right)
$$


where

$$
v=\left(A_{00}, \ldots, A_{0 i}, \ldots\right)
$$

The quasi-diagonal backpropagated metric is the quasi-diagonal metric obtained from the backpropagated metric. The quasi-diagonal Fisher metric is the one obtained from the unitwise Fisher metric. The quasi-diagonal OP metric is the one obtained from the unitwise OP metric.

The reasoning in this section can be summarized as follows.

Proposition 24. Assume that the activation function is a fixed nonlinear function composed with an affine function. Then the quasi-diagonal reduction $\tilde{A}$ of an intrinsic metric $A$ is intrinsic.

Importantly, the matrix $\tilde{A}=\operatorname{diag}(A)+A_{00}^{-1}(v \otimes v)-\operatorname{diag}\left(A_{00}^{-1}(v \otimes v)\right)$ is the sum of a diagonal matrix and a rank-1 matrix. This allows for easy inversion, resulting in a quasi-diagonal inverse matrix.

Proposition 25 (QUASI-Diagonal Gradient STeP). Let $\tilde{A}$ be the quasi-diagonal reduction of $A$. Let $b=\left(b_{0}, \ldots, b_{i}, \ldots\right)$ and $w=\tilde{A}^{-1} b$. Then $w$ is given by

$$
\begin{aligned}
w_{i} & =\frac{b_{i} A_{00}-b_{0} A_{0 i}}{A_{i i} A_{00}-A_{0 i}^{2}} \quad \text { for } i \neq 0 \\
w_{0} & =\frac{b_{0}}{A_{00}}-\sum_{i \neq 0} \frac{A_{0 i}}{A_{00}} w_{i}
\end{aligned}
$$

Thus, only the entries $A_{00}, A_{i i}$ and $A_{0 i}$ of the original matrix $A$ need to be known in order to implement gradient descent using the quasi-diagonal metric defined by $\tilde{A}$.

Note that if the original matrix $A$ is positive-definite, we have $A_{00}>0$ and $A_{00} A_{i i}>A_{0 i}^{2}$ (by the Cauchy-Schwarz inequality applied to the first and $i$-th basis vectors), so that the solution $w$ above is well-defined and unique.

\subsection{Intrinsic gradients}

Thanks to these intrinsic metrics we can define intrinsic gradient directions in parameter space. Given a dataset $\mathcal{D}$ of inputs $x$ and corresponding targets $y$, the average loss function is

$$
L_{\theta}:=\mathbb{E}_{x \in \mathcal{D}} \ell_{\theta}(y)
$$

where we put a subscript $\theta$ to make explicit its dependency on the parameters of the network. Given an intrinsic metric $\|\cdot\|$, the differential

$$
G=-\frac{\partial L_{\theta}}{\partial \theta}
$$


of the average loss with respect to the full parameter set $\theta$, defines a gradient direction $\nabla_{\theta} L$ by the usual definition: it is the only tangent vector such that for any $\delta \theta$ we have

$$
L_{\theta+\delta \theta}=L_{\theta}+\left\langle\nabla_{\theta} L, \delta \theta\right\rangle+O\left(\|\delta \theta\|^{2}\right)
$$

where $\langle\cdot, \cdot\rangle$ is the scalar product associated with the norm $\|\cdot\|$. In a parametrization where $\|\cdot\|^{2}$ is given by a symmetric, positive definite matrix $A$, the gradient is given by

$$
\nabla_{\theta} L=A^{-1} \frac{\partial L}{\partial \theta}=-A^{-1} G
$$

The gradient $\nabla_{\theta} L$ is an intrinsic tangent vector on the parameter set.

Definition 26. The natural gradient, unitwise natural gradient, backpropagated metric gradient, $O P$ gradient, unitwise $O P$ gradient, and their quasi-diagonal reductions, respectively, are the following update rule for $\theta$ :

$$
\theta \leftarrow \theta-\eta \nabla_{\theta} L
$$

where $\nabla_{\theta} L=A^{-1} \frac{\partial L}{\partial \theta}$ is the gradient of the average loss function $L$ computed with $A$ the natural metric, unitwise natural metric, backpropagated metric, $O P$ metric, unitwise $O P$ metric, and their quasi-diagonal reductions, respectively.

The algorithms of Section 1 are the application of these updates to ordinary neural networks, written out with $[0 ; 1]$-valued activities and sigmoid activation function. More details on how this works out are given below (Section 2.5).

This update is intrinsic only under all affine reparametrizations of the parameter $\theta$. Indeed, even if the tangent vector $\nabla_{\theta} L$ giving the direction of the gradient is fully intrinsic, adding a tangent vector to a parameter $\theta$ is not an intrinsic operation (if two parametrizations differ by a non-affine transformation, then the additions will not amount to the same).

On the other hand, the ideal limit when the learning rate $\eta$ tends to 0 is intrinsic: the trajectories of the differential equation

$$
\frac{\mathrm{d} \theta(t)}{\mathrm{d} t}=-\nabla_{\theta(t)} L
$$

are intrinsic trajectories in parameter space for the unitwise natural gradient and backpropagated metric.

For the quasi-diagonal algorithms, invariance is always restricted to affine reparametrizations, since this is the setup in which they are well-defined. 


\subsection{The Fisher matrix for neural networks}

The general definitions above depend on the choice of a metric on the output $\omega$ of the network. When this metric is the Fisher metric on the output layer, applying the general definitions above to ordinary neural networks leads to the algorithms of Section 1. This is mostly by direct computation and we do not reproduce it fully. Let us however discuss the Fisher metric in more detail.

For each input $x$, the network defines a probability distribution $\omega$ on the outputs $y$. This probability distribution depends on the parameters of the network. Thus, for each input $x$, we can define a datum-wise Fisher matrix on the parameter set:

$$
F(x)_{w_{i j} w_{i^{\prime} j^{\prime}}}=\mathbb{E}_{y \mid x} \frac{\partial \ell(y)}{\partial w_{i j}} \frac{\partial \ell(y)}{\partial w_{i^{\prime} j^{\prime}}}
$$

where as above $\ell(y)=\ln \omega(y)$ and where $\mathbb{E}_{y \mid x}$ denotes expectation for $y$ following the distribution $\omega$ defined by the input $x$.

The dataset together with the network define a probability distribution on pairs $(x, y)$, by first choosing at random an input $x$ in the dataset, then running the network on this input. The Fisher matrix associated with this distribution on pairs $(x, y)$ is the average of the datum-wise Fisher matrix over the dataset

$$
F=\mathbb{E}_{x \in \mathcal{D}} F(x)
$$

(see [AN00, Section 8.2), or more explicitly

$$
F_{w_{i j} w_{i^{\prime} j^{\prime}}}=\mathbb{E}_{x \in \mathcal{D}} \mathbb{E}_{y \mid x} \frac{\partial \ell(y)}{\partial w_{i j}} \frac{\partial \ell(y)}{\partial w_{i^{\prime} j^{\prime}}}
$$

Exact Fisher matrix versus one-sample Fisher matrix. One possible way to train neural networks using the natural gradient is to estimate the Fisher matrix by taking an input $x$ in the dataset, taking a random output $y$ for this input, and add the term $\frac{\partial \ell(y \mid x)}{\partial w_{i j}} \frac{\partial \ell(y \mid x)}{\partial w_{i^{\prime} j^{\prime}}}$ to the current estimate of the Fisher matrix (with a discount factor for older contributions in an online setting). This leads to the Monte Carlo natural gradient with $K$ samples $y$ per input $x$ :

$$
\hat{F}_{w_{i j} w_{i^{\prime} j^{\prime}}}=\mathbb{E}_{x \in \mathcal{D}} \frac{1}{K} \sum_{k=1}^{K} \frac{\partial \ell\left(y_{k}\right)}{\partial w_{i j}} \frac{\partial \ell\left(y_{k}\right)}{\partial w_{i^{\prime} j^{\prime}}}
$$

where each $y_{k}$ is drawn according to the output probability distribution $\omega$ defined by the output for input $x$. Even $K=1$ can lead to reasonable results (Section 4).

An important variant uses for $y$ the actual target value for input $x$, instead of taking $y$ as a random sample given by the activations of the 
output layer:

$$
\hat{F}_{w_{i j} w_{i^{\prime} j^{\prime}}}=\mathbb{E}_{x \in \mathcal{D}} \frac{\partial \ell(y(x))}{\partial w_{i j}} \frac{\partial \ell(y(x))}{\partial w_{i^{\prime} j^{\prime}}}
$$

with $y(x)$ the target for $x$ : this is just the outer product (OP) metric of Definition 14. It is not an unbiased estimate of the Fisher metric; still, hopefully when the network converges towards the desired targets, the law of $y$ taken from the output distribution converges to the actual target and the two variants get close. This variant has been present for a long time in studies on natural gradient (as is clear, e.g., from Equation (14) in [APF00]) and is elaborated upon in [RMB07]. As pointed out in [PB13], the two variants are often confused.

These two variants are both intrinsic. The OP variant, contrary to the true natural gradient, depends on the targets and not only on the network and inputs.

Both the true natural gradient, its Monte Carlo approximation, and its "one-sample"/OP variant give rise to a unitwise version and to a quasidiagonal version (Section 2.3). For a network with $n$ units and at most $d$ incoming connections per unit, the algorithmic cost of processing each data sample is $O\left(K n^{2} d^{2}\right)$ for the Monte Carlo natural gradient, $O\left(K n d^{2}\right)$ for its unitwise version and $O(K n d)$ for its quasi-diagonal reduction. Algorithmic cost for the OP metric is the same with $K=1$.

In Section 4 we compare performance of the unitwise natural gradient, Monte Carlo unitwise natural gradient with $K=1$, and unitwise OP natural gradient. We will see that although the $\mathrm{OP}$ metric and the one-sample $(K=1)$ Monte Carlo natural gradient look similar, the latter can perform substantially better.

Exact Fisher matrix computation. It is possible compute the exact Fisher matrix (rather than using a single value for $y$ ) by using the Fisher modulus and backpropagation transfer rates. The latter can be computed by doing $n_{\text {out }}$ backpropagations for each input. This is of course more convenient than computing the expectation $\mathbb{E}_{y \mid x}$ by summing over the (in the Bernoulli case) $2^{n_{\text {out }}}$ possible outcomes for $y$.

The backpropagation transfer rates $J_{k}^{k_{\text {out }}}$ from Definition 2 simply implement the general Definition 19 for ordinary neural networks. In Section 1, the unitwise natural gradient was obtained from these transfer rates through the Fisher modulus. Here we reproduce the corresponding formula for all terms of the Fisher matrix, not only the terms of the unitwise Fisher matrix incoming to a given unit, so we introduce a Fisher modulus indexed by pairs of units.

Proposition 27 (ExaCt Fisher matrix for NeUral Networks). Let $x$ be an input for the network. Compute the transfer rates $J_{k}^{k_{\text {out }}}$ as in 
Definition 2. Depending on output layer interpretation, set for each pair of units $k$ and $k^{\prime}$ :

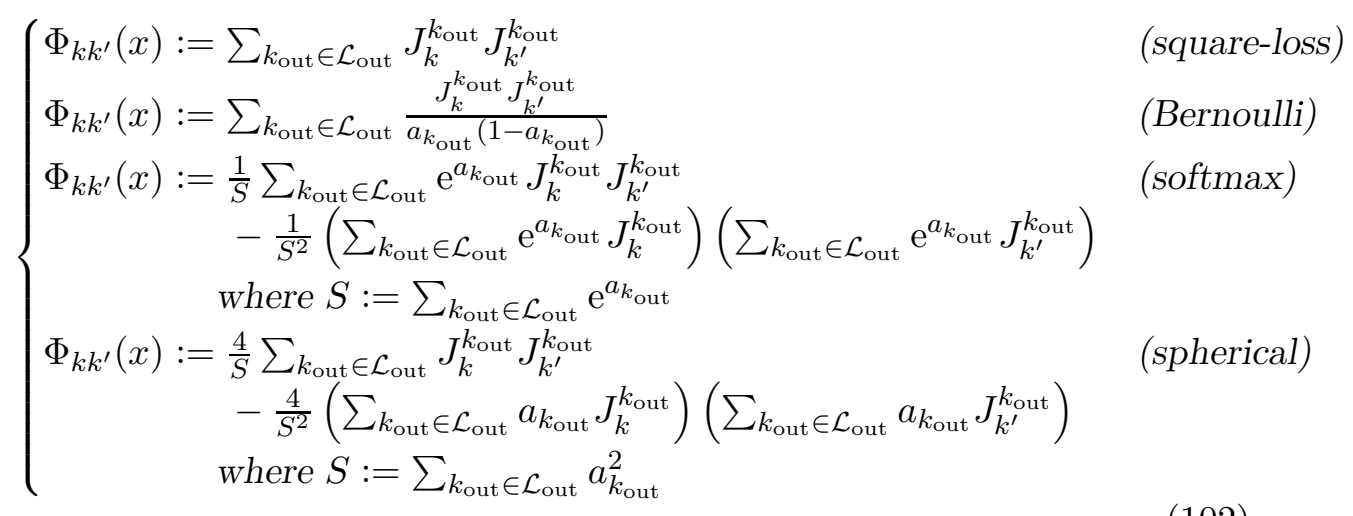

Then the entry of the datum-wise Fisher matrix $F(x)$ associated with parameters $w_{i k}$ and $w_{j k^{\prime}}$ (including biases with $i=0$ or $j=0$ ) is

$$
F(x)_{w_{i k} w_{j k^{\prime}}}=a_{i} a_{j} r_{k} r_{k^{\prime}} \Phi_{k k^{\prime}}
$$

and thus the corresponding entry in the Fisher matrix is

$$
F_{w_{i k} w_{j k^{\prime}}}=\mathbb{E}_{x \in \mathcal{D}} a_{i} a_{j} r_{k} r_{k^{\prime}} \Phi_{k k^{\prime}}
$$

The proof is given in the Appendix and is a more or less straightforward application of the results of the previous section, together with an explicit computation of the Fisher metric on the output in the Bernoulli, square-loss, and classification interpretations.

So it is possible to compute the full Fisher matrix by performing $n_{\text {out }}$ independent backpropagations for each sample input. The Fisher matrix $F$, being the average of $F(x)$ over the dataset, may be approximated by the standard online or small-batch techniques using samples from the dataset.

For a network with only one hidden layer, this simplifies and no additional backpropagations are needed. Indeed, the backpropagation transfer rates of the input layer are never used, and on the hidden layer are given by

$$
J_{k}^{k_{\text {out }}}=w_{k k_{\text {out }}} r_{k_{\text {out }}}
$$

from which the Fisher modulus can be immediately computed. This is the case treated in Kur94 (for the Bernoulli interpretation).

\section{Some properties of unitwise algorithms and their quasi-diagonal approximations}

\subsection{Performance improvement at each step}

A common feature of all gradient-based algorithms in any metric is that the objective function improves at each step provided the learning rate is 
small enough. Consequently this holds for the unitwise natural gradient, backpropagated metric gradient, and their quasi-diagonal reductions.

Proposition 28. Suppose that training has not reached a local optimum, i.e., that the gradient vector $G$ of Section 1 does not vanish. Suppose that the metric considered is non-degenerate (i.e., respectively, that the matrices $F^{(k)}$ or $M^{(k)}$ are invertible, or that the denominators in the quasidiagonal algorithms do not vanish), so that the algorithms considered are well-defined. Suppose that the chosen interpretation $\omega$ of the output layer depends smoothly on the output layer activities.

Then there exists a value $\eta_{C}$ of the learning rate such that, for any learning rate $\eta<\eta_{C}$, the value of the loss function strictly decreases after one step of the unitwise natural gradient, backpropagated metric gradient, or their quasi-diagonal reductions.

As usual, the value of $\eta_{C}$ depends on the current state of the network and thus may change over the course of training.

\subsection{Invariance properties}

The algorithms presented in Section 1 are the implementation of the gradients and metrics defined in Section 2, written out using [0;1]-valued activities and the logistic activation function. We could have written them out, for instance, using $[-1 ; 1]$-valued activities and the tanh activation function, and the learning trajectory would be the same - provided, of course, that the initialization was done so that both implementations of the network behave the same at startup. We present a more precise formulation of this property.

Imagine that the inputs of the network are subjected to simple transformations such as scaling ( $a_{i} \leftarrow \alpha_{i} a_{i}$ for $i$ in the input layer) or $0 / 1$ inversion $\left(a_{i} \leftarrow 1-a_{i}\right)$. There is a simple way to change the parameters of subsequent units so that the final activation of the network stays the same, namely, $w_{i j} \leftarrow w_{i j} / \alpha_{i}$ for scaling and $w_{i j} \leftarrow-w_{i j}, w_{0 j} \leftarrow w_{0 j}+w_{i j}$ for $0 / 1$ inversion. So clearly the expressivity of a neural network is not sensitive to such changes.

However, training will behave differently. For instance, if we apply one step of backpropagation training to the scaled inputs with the scaled network, the coefficients of units which have been scaled down $\left(\alpha_{i}<1\right)$ will evolve more slowly and conversely for $\alpha_{i}>1$. The final output of the network after the update will be different. (Hence the common practice of rescaling the activities of units.) The same goes for $0 / 1$ inversion in a slightly more complicated way: evolution of the bias depends on the activity of input units, and the weights from input units with activity close to 0 will evolve faster than those with activity close to 1, as seen on Figure 1.

We would like the following invariance for a training procedure: If we start with two networks $N$ and $N^{\prime}$ which are fed inputs $x$ and $x^{\prime}$ with $x^{\prime}$ 
obtained from a simple transformation of $x$, and if the parameters of $N^{\prime}$ are set such that initially its output is the same as $N$, then we would like the outputs of $N$ and $N^{\prime}$ to stay the same after one step of the training procedure.

This is not satisfied by backpropagation. However, for any affine transform of the activities of any unit, this is satisfied by the natural gradient, unitwise natural gradient, unitwise outer product gradient, backpropagated metric gradient, and their quasi-diagonal reductions.

The sigmoid and tanh networks correspond to each other by the following rewriting, thanks to $\tanh (x)=2 \operatorname{sigm}(2 x)-1$ : if $a_{k}=\operatorname{sigm}\left(\sum_{i \rightarrow k} w_{i k} a_{i}+\right.$ $\left.w_{0 k}\right)$ and $a_{k}^{\prime}=\tanh \left(\sum_{i \rightarrow k} w_{i k}^{\prime} a_{i}^{\prime}+w_{0 k}^{\prime}\right)$ (and interpretation of the output layer in the tanh case is done by putting back the activities in $[0 ; 1]$ via $\left.a^{\prime} \mapsto 1 / 2+a^{\prime} / 2\right)$, then the two networks will behave the same if we set $w_{i k}=4 w_{i k}^{\prime}(i \neq 0)$ and $w_{0 k}=2 w_{0 k}^{\prime}-2 \sum_{i \neq 0} w_{i k}^{\prime}$.

Definition 29. Let $k$ be an input or internal unit. Call $(\alpha, \beta, \gamma)$-affine reparametrization of unit $k$ the following operation: Replace the activation of unit $k$

$$
a_{k}=f_{\theta_{k}}^{k}\left(a_{i_{1}}, \ldots, a_{i_{n_{k}}}\right)
$$

where $\theta_{k}=\left(w_{0 k},\left(w_{i k}\right)_{i \rightarrow k}\right)$, with

$$
a_{k}^{\prime}=\alpha f_{\gamma \theta_{k}^{\prime}}^{k}\left(a_{i_{1}}, \ldots, a_{i_{n_{k}}}\right)+\beta
$$

where $\theta_{k}^{\prime}=\left(w_{0 k}^{\prime},\left(w_{i k}^{\prime}\right)_{i \rightarrow k}\right)$. Send $a_{k}^{\prime}$ instead of $a_{k}$ to the next layer of the network, with weights modified as follows:

$$
w_{k j}^{\prime}=w_{k j} / \alpha, \quad w_{0 j}^{\prime}=w_{0 j}-w_{k j} \beta / \alpha
$$

for all units $j$ such that $k \rightarrow j$, and $w_{i k}^{\prime}=w_{i k} / \gamma$ for all units $i$ with $i \rightarrow k$ (including $i=0$ ), so that the final outputs before and after the reparametrization are the same.

The passage from sigm to tanh consists in applying the $(2,-1,2)$-reparametrization to all units. We have restricted the definition to non-output units to simplify notation; for output units a corresponding reparametrization of the output interpretation has to be done.

The following result is an immediate consequence of the intrinsic definition of the algorithms. It is only part of the invariance properties of the objects from Section 2, In particular, in the limit of small learning rates $(\eta \rightarrow 0)$, the trajectories (96) of the unitwise natural gradient, backpropagated metric gradient, and unitwise OP gradient, are invariant under all smooth one-to-one reparametrization and not only affine ones. 
Proposition 30 (INVARIANCE UNDER AFFine RePARAMETRIzATION OF ACTIVITIES). Consider a network obtained from an initial network by applying any number of $(\alpha, \beta, \gamma)$-affine reparametrizations to any number of units (where $\alpha, \beta$ and $\gamma$ may depend on the unit).

Then, after one step of the unitwise natural gradient, backpropagated metric gradient, Monte Carlo unitwise natural gradient, unitwise OP gradient, or their quasi-diagonal reductions, the final outputs of the non-reparametrized and reparametrized networks are the same.

Consequently, the learning trajectories, and performance, of the two networks with these corresponding initializations are the same.

This may seem a simple thing, but we should keep in mind that this property is not satisfied by backpropagation, or by quasi-Newton methods if the latter use diagonal approximations of the Hessian.

In particular, the algorithms presented here are insensitive to shifting and scaling of all units in the network. Traditionally, it is recommended to normalize the activities on input units so that they average to 0 over the dataset and have a prescribed variance: the algorithms here automatically do the same in an implicit way, for all (not only input) units. As a consequence, units with low activation levels get updated as fast as highly activated units. (Note that as discussed after the definition of the quasi-diagonal algorithms, these averages and variances are computed according to non-uniform weights on the dataset given by the Fisher modulus or backpropagated modulus.)

Still the invariance above only applies if the two networks considered have corresponding initializations. For instance, if the initial weights are random with a variance set to 1 whatever the data, obviously the initial behavior of the network will be sensitive to scaling of its input. So these methods do not remove the need for traditional recommendations for initializing the weights (either by normalizing the data and then taking weights of size 1 , or by taking initial weights depending on the variance or covariance matrix of the data).

The unitwise gradients (natural, backpropagated metric, OP, and Monte Carlo natural), but not their quasi-diagonal reductions, have a further, more interesting invariance property: invariance under affine recombination of the signals a unit receives from its various incoming units. For instance, if we start with zero weights, an internal unit will evolve in the same way if it receives $f$ and $f+\varepsilon g$ (where $f$ and $g$ are seen as function of the input $x$ ) as if it receives $f$ and $g$. This is especially useful if $g$ is correlated to the desired output.

Proposition 31 (INVARIANCE UNDER AFFINE RECOMBINATION OF INCOMING SignALs). Consider a neural network and define a new one in the following way. Let $k$ be a non-input unit in the network, with $n_{k}$ 
incoming units, and let $\varphi: \mathbb{R}^{k} \rightarrow \mathbb{R}^{k}$ be an invertible affine map. Define a new network by replacing the activation function at unit $k$

$$
a_{k}=f_{\theta_{k}}^{k}\left(a_{i_{1}}, \ldots, a_{i_{n_{k}}}\right)
$$

with

$$
a_{k}=f_{\varphi^{*}\left(\theta_{k}\right)}^{k}\left(\varphi\left(a_{i_{1}}, \ldots, a_{i_{n_{k}}}\right)\right)
$$

still parametrized by $\theta_{k}$, where $\varphi^{*}\left(\theta_{k}\right)$ results from applying the duat10 inverse affine transformation $\varphi^{*}$ to $\theta_{k}$, so that initially the responses of the original and reparametrized networks are the same.

Then, after one step of the unitwise natural gradient, backpropagated metric gradient, Monte Carlo natural gradient, or unitwise OP gradient, with respect to $\theta_{k}$, the final outputs of the non-reparametrized and reparametrized networks are the same.

Consequently, the learning trajectories, and performance, of the two networks with these corresponding initializations are the same.

Once more, this is not simply $\varphi$ in one place cancelling out $\varphi^{-1}$ in another: indeed, backpropagation or quasi-Hessian methods do not have this property, and neither do the quasi-diagonally-reduced algorithms.

Proof. This comes as a consequence of the best-fit interpretation (Proposition 32) below.

It also follows from the intrinsic constructions by noting that, unlike the quasi-diagonal reductions, the construction of these gradients never breaks down the $n_{k}$-tuple of incoming activities into its components from each incoming unit; thus, contrary to the quasi-diagonal reductions, we could have written the unitwise natural and backpropagated metrics in a setting where activation functions are given by $a_{k}=f_{\theta_{k}}^{k}\left(g\left(a_{i_{1}}, \ldots, a_{i_{n_{k}}}\right)\right)$ where $g$ is a fixed, parameterless map with values in a manifold.

\subsection{Best-fit interpretation}

The unitwise natural gradient, backpropagated metric gradient, and unitwise OP gradient (but not their quasi-diagonal reductions) share an interpretation as a least-squares regression problem at each unit. Namely, the backpropagated value $b_{k}(x, y)$ on input $x$ and target $y$ indicates how the activity of unit $k$ should change on input $x$. Seeing $b_{k}$ as a function of the input $x$, unit $k$ has to use the activities of incoming units $i$ (also seen as functions of $x$ ) and combine them using the weights $w_{i k}$, to match $b_{k}(x, y)$ as close as possible for each $x$. This idea is presented in [Kur94 in a more

\footnotetext{
${ }^{10} \theta_{k}$ is an affine form over the $n_{k}$-tuple of incoming activities. $\varphi^{*}$ is defined, axiomatically, by the property that applying $\varphi^{*}\left(\theta_{k}\right)$ to the activities transformed by $\varphi$, is the same as applying $\theta_{k}$ to the untransformed activities. Decomposing $\theta_{k}=\left(w_{0 k},\left(w_{i k}\right)_{i \rightarrow k}\right)$, the affine matrix defining $\varphi^{*}$ is the transpose of the inverse of the affine matrix defining $\varphi$.
} 
specific setting. This is also relevant to the behavior of the algorithms when the matrices $F$ and $M$ defining the metrics are singular or close to singular, as we will see.

Proposition 32 (Intrinsic gradients as Best fit to $b$ ). Let $k$ be a non-input unit in the network. For $x$ in the dataset $\mathcal{D}$, let $b_{k}(x)$ be the backpropagated value (4) obtained on input $x$ and the corresponding target $y$.

Consider the solution $\lambda=\left(\lambda_{i}\right)$ to the following weighted least-squares problem:

$$
\lambda=\arg \min \left\{\sum_{x \in \mathcal{D}}\left(\sum_{i} \lambda_{i} a_{i}(x)-\frac{b_{k}(x)}{r_{k}(x) \Phi_{k}(x)}\right)^{2} W_{x}\right\}
$$

where $i$ runs over the incoming units to $k$ (including $i=0$ with $a_{0} \equiv 1$ ), $\Phi_{k}(x)$ is the Fisher modulus (Definition [3), and the weights are

$$
W_{x}:=r_{k}(x)^{2} \Phi_{k}(x)
$$

Then the unitwise natural gradient step (21) is given by $\lambda$, namely, the update is $w_{i k} \leftarrow w_{i k}+\eta \lambda_{i}$ at each unit $k$.

The same holds for the backpropagated metric gradient using the backpropagated modulus $m_{k}$ (Definition [7) instead of the Fisher modulus $\Phi_{k}$.

The same holds for the unitwise OP gradient using $b_{k}(x)^{2}$ instead of the Fisher modulus $\Phi_{k}$.

Thus, the gradient step depends on the linear span of the incoming activities $\left(a_{i}(x)\right)_{i \rightarrow k}$, seen as functions over the dataset (which, by the way, proves Proposition 31 above). This is why the gradient step is the same whether the unit receives signals $f(x)$ and $g(x)$ or $f(x)$ and $f(x)+\varepsilon g(x)$. Thus, these algorithms perform an implicit orthonormalization of the incoming signals at each unit (not only input units) in the network.

Proof. A direct application of the well-known formula for the solution of the weighted least-squares problem (111), with the choice of weight (112), yields exactly the updates (20) and (30).

Non-invertibility and regularization of the matrices. In several situations the matrices $F$ and $M$ used to define the unitwise natural update and backpropagated metric update can be singular.

This is the case, for instance, if one input unit is uniformly set to 0 over all elements in the dataset: obviously such a unit is not informative, and the corresponding term will vanish both in the metric and in the gradient. This is also the case when, e.g., two units incoming to the same unit are 
perfectly correlated. Correlation in the activation profiles happens systematically in case the size of the dataset is smaller than the number of incoming parameters at a given unit.

The linear regression viewpoint limits, in theory, the seriousness of these issues: this only means the linear regression problem has several solutions (one can add any quantity to a non-informative weight), and any of them will do as an update. Indeed, for instance, the matrix $M$ in Definition 9 is of the form $A A^{\top}$, and $M^{-1}$ is applied to the vector $G$ which is of the form $A Y$, thus $G$ always lies in the image of $A$ and thus the linear system is underdetermined, not overdetermined. From the gradient ascent viewpoint this means the matrix $M$ will be singular but the gradient term $\partial L / \partial w$ will vanish in the corresponding directions.

Numerically, however, the issue must be dealt with. One can use the Moore-Penrose pseudoinverse of $M$ (or $F$ in the Fisher matrix case), obtained by adding $\varepsilon$. Id to $M$ or to $F$ with very small $\varepsilon$. This is a standard regularization technique. It has the advantage of producing a well-defined update when $\varepsilon \rightarrow 0$, asymptotically independent of $\varepsilon$.

Thus, if a formal definition is needed, one can decide to use the MoorePenrose pseudoinverse for $M^{-1}$ and $F^{-1}$ in the definition of the updates. However, this formally breaks the invariance properties: the Moore-Penrose pseudoinverse selects, among the several possible solutions $\left(\lambda_{i}\right)$ to an underdetermined least squares problem, the one with smallest norm $\sum \lambda_{i}^{2}$, and this is not intrinsic.

\section{A first experimental comparison}

Although the main focus of this article is theoretical, we performed a light set of experiments to ensure that the suggested methods are viable. The companion article Oll13 contains more in-depth experiments with recurrent networks and complex symbolic sequences.

To test the influence of the different methods, we chose a very simple problem in which a perfect solution is expected to be found. A sparsely connected network with 5 layers of size 100, 30, 10, 30, and 100 was built, and 16 random length-100 binary strings were fed to the input layer, with the target equal to the input (auto-encoding). Ideally the network learns to encode each of the 16 samples using 4 bits on the middle layer (thus with room to spare) and uses the bottom layer parameters to rewrite the output from this. This is purely an optimization task without a learning aspect, as there is no generalization to be done and no underlying pattern. The focus is on which methods are able to converge to a good solution.

The sparsely connected network is built at random in each instance as follows. Each of the 100 units in the input layer is linked to 5 randomly selected nodes in the first hidden layer. Each of the 30 units in the first 
hidden layer is linked to 5 random nodes in the middle hidden layer. The scheme is reversed for the bottom part of the model: each of the 100 output units is linked to 5 random nodes in the last hidden layer, and each unit in the last hidden layer is linked to 5 random nodes of the middle hidden layer.

For each instance, the dataset is made of 16 random binary strings of length 100. The target for each input is identical to the input. We use Bernoulli interpretation of the output.

Note that this setting is adverse for the unitwise and quasi-diagonal natural gradients, which require a small output layer; this must be remembered in the comparisons below.

To test the influence of parametrization on non-invariant algorithms, and to check invariance of the invariant ones, each algorithm was implemented both using $\operatorname{sigm}(x)$ and $\tanh (x)$ as the activation function.

The methods tested are: backpropagation; unitwise natural gradient; quasi-diagonal natural gradient; unitwise OP gradient; Monte Carlo unitwise or quasi-diagonal natural gradient with one sample $(K=1$ in (100) $)$; backpropagated metric gradient; quasi-diagonal backpropagated metric gradient; diagonal Gauss-Newton ([LBOM96, SZL13]; equivalent to keeping only the diagonal terms in the quasi-diagonal backpropagated metric gradient); and a batch version of Adagrad/RMSprop [DHS11] in which the learning rate for each gradient component is divided by the root mean square of this component over the samples.

Since the sample size is small, the algorithms were run in batch mode.

Regularization. The algorithms were taken directly from Section 1) To all methods except backpropagation, we added a regularization term of $10^{-4}$ Id to the various matrices involved, to stabilize numerical inversion. This value is not so small; values such as $10^{-7}$ seemed to affect performance. This is probably due to the small sample size (16 samples): each sample contributes a rank-1 matrix to the various metrics. Larger sample sizes would probably need less regularization.

Initialization. For the tanh activation function, all the weights were initialized to a centered Gaussian random variable of standard deviation $1 / \sqrt{d_{j}}$ with $d_{j}$ the number of units pointing to unit $j$, and the biases set to 0 . For the sigmoid activation function, the initialization was the corresponding one (using Eqs. 46] and 47) so that initially the responses of the networks are the same: namely, each weight was set to a centered Gaussian random variable of standard deviation $4 / \sqrt{d_{j}}$, and then the bias at unit $k$ was set to $-\sum_{i i \rightarrow k} w_{i k} / 2$. This initialization has the property that if the incoming signals to a unit are independent, centered about $1 / 2$ (sigmoid) or 0 (tanh) and of variance $\sigma$ with $\sigma$ not too large, then the output of the unit is also centered of variance $\approx \sigma$. (The factor 4 in the sigmoid case compensates 
for the derivative $1 / 4$ of the sigmoid function at 0 .) See the argument in GB10 11.

Learning rate. A simple adaptive method was used for the learning rate. All methods based on gradients in a metric have a guarantee of improvement at each step if the learning rate is small enough. So in the implementation, if a step was found to make the loss function worse (in a batch mode, thus summed over all samples), the step was cancelled and the learning rate was divided by 2 . If the step improves the loss function, the learning rate is increased by a factor 1.1. The initial learning rate was set to 0.01 ; in practice the initial value of the learning rate is quickly forgotten and has little influence.

Unfortunately this scheme only makes sense in batch mode, but it has the advantage of automatically selecting learning rates that suit each method, thus placing all methods on an equal footing.

Execution time and number of iterations. First, 10, 000 steps of backpropagation were performed on the whole dataset, in batch mode. The resulting running time was set aside and converted to an equivalent number of iterations for all of the other algorithms. This is a very rough way to proceed, since the running times can depend on the implementation details, 12 , and vary from run to run (because floating point operations do not take the same time depending on the numbers they are operating on, especially when both very small and very large values are involved).

Most of all, the different methods scale in different ways with the network, and so the network used here may not be representative of other situations. In particular this auto-encoder setting with 100 output units puts the unitwise natural gradient and quasi-diagonal natural gradient at a disadvantage (on the same time budget they must performe $n_{\text {out }}$ backpropagations per sample), compared to, e.g., a classification setting.

Nevertheless, we give in Table 4the numbers of iterations giving roughly equal running time for each method.

The natural gradient was also tested (using the exact full Fisher matrix as obtained from Proposition 27). The computational cost is very high and only 10 iterations take place in the alloted time, too few for convergence. Thus we do not report the associated results.

Results. In Table 2, we report the average loss per sample, in bits, and its standard deviation, at the end of the allocated number of training iterations. These values can be interpreted as representing the average number of bits

\footnotetext{
${ }^{11}$ The other initialization suggested in GB10], with weights of magnitude $\sqrt{6 /\left(d_{j}+d_{j}^{\prime}\right)}$ with $d_{j}^{\prime}$ the number of edges from $j$, did not make any significant difference in our setup.

${ }^{12}$ We tried to implement each method equally carefully.
} 


\begin{tabular}{lc}
\multicolumn{1}{c}{ Method } & Number of iterations \\
\hline Backpropagation (sigmoid) & 10,000 \\
Backpropagation (tanh) & 10,000 \\
Natural gradient & 9 to 10 \\
Unitwise natural gradient & 2,100 to 2,300 \\
Quasi-diagonal natural gradient & 2,800 to 3,100 \\
Backpropagated metric & 4,200 to 4,300 \\
Quasi-diagonal backpropagated metric & 7,400 to 7,500 \\
Monte Carlo unitwise natural gradient $(K=1)$ & 2,800 to 2,900 \\
Monte Carlo quasi-diagonal natural gradient $(K=1)$ & 3,900 to 4,000 \\
Unitwise OP gradient & 4,000 to 4,100 \\
Diagonal Gauss-Newton & 7,700 to 7,800 \\
AdaGrad & 8,000 to 8,100 \\
\hline
\end{tabular}

Table 1: Number of iterations resulting in approximately equal execution times for our problem

of an output that the model did not learn to predict correctly (out of 100). The results of the implementations using sigmoid and tanh activation are reported separately.

Performance as a function of time is plotted in Figure 3 ,

The statistics were made using 20 independent runs for each method.

Interpretation. These results are mainly illustrative: the situation considered here may not be representative because of the small sample size and network size involved.

Still, it is clear that for problems of this size, the more elaborate algorithms are very competitive. Only the tanh implementation of the diagonal Gauss-Newton method comes close to the invariant algorithms (while its performance in sigmoid implementation is not as good).

As can be expected, the invariant algorithms have similar performance in sigmoid or tanh implementation: trajectories match each other closely (Figure 4). The variations are caused, first, by random initialization of the dataset and weights in each run, and second, by the inclusion of the regularization terms $\varepsilon \mathrm{Id}$, which breaks invariance. If the effect of the latter is isolated, by having the same initialization in tanh and sigmoid implementations, the trajectories coincide for the first few iterations but then start to differ, without affecting overall performance.

In this setting, the natural gradient, in its unitwise and quasi-diagonal versions, seems to perform slightly worse than the backpropagated metric methods. This might be an effect of the large output layer size (which directly affects their computational complexity) combined with a given com- 


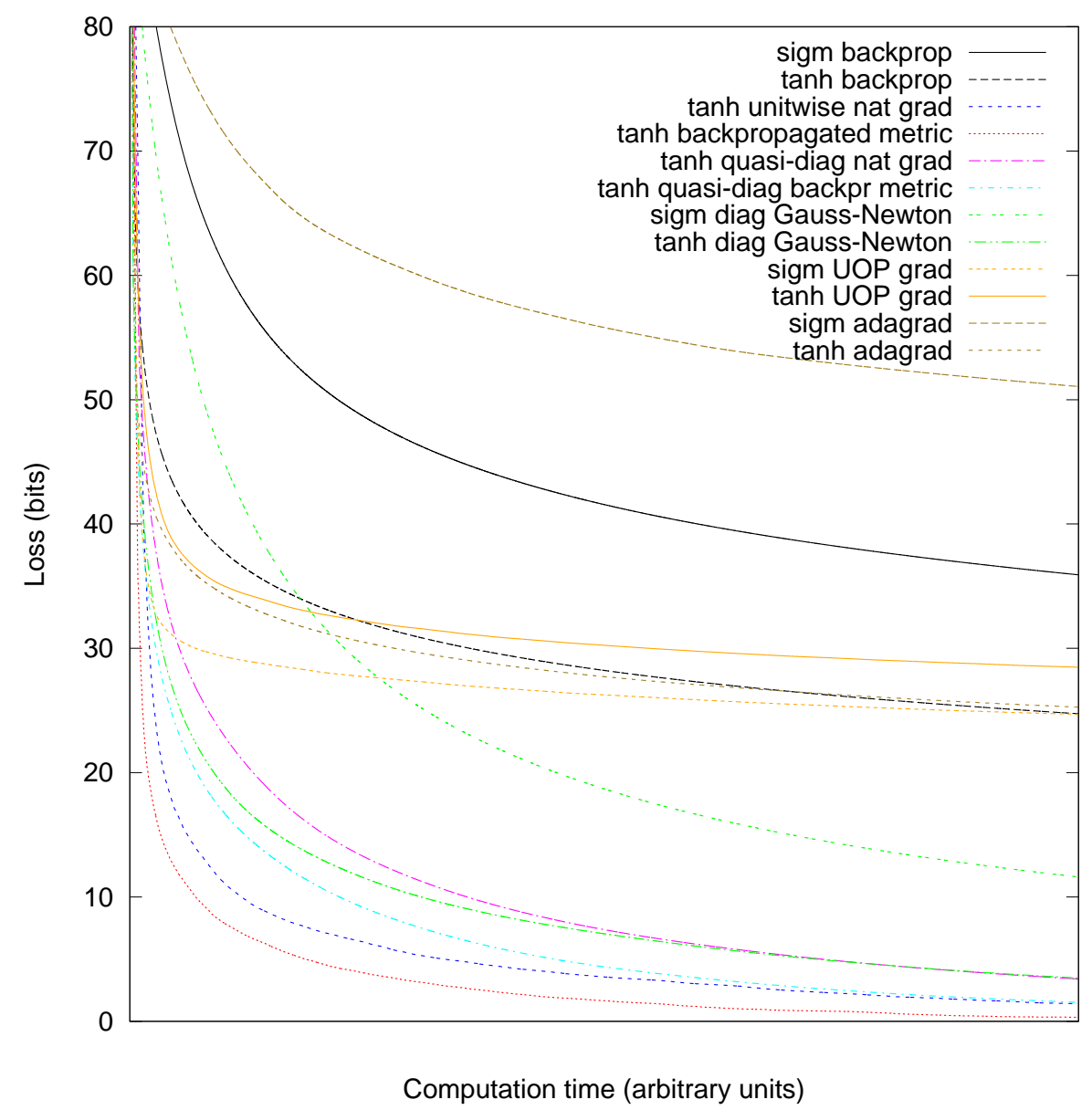

Figure 3: Performance over time of all algorithms involved. For better readability the trajectories of the invariant algorithms have been plotted only in tanh implementation (Figs. 2 and 4 show them in sigmoid implementation for completeness). Average over 20 runs. 

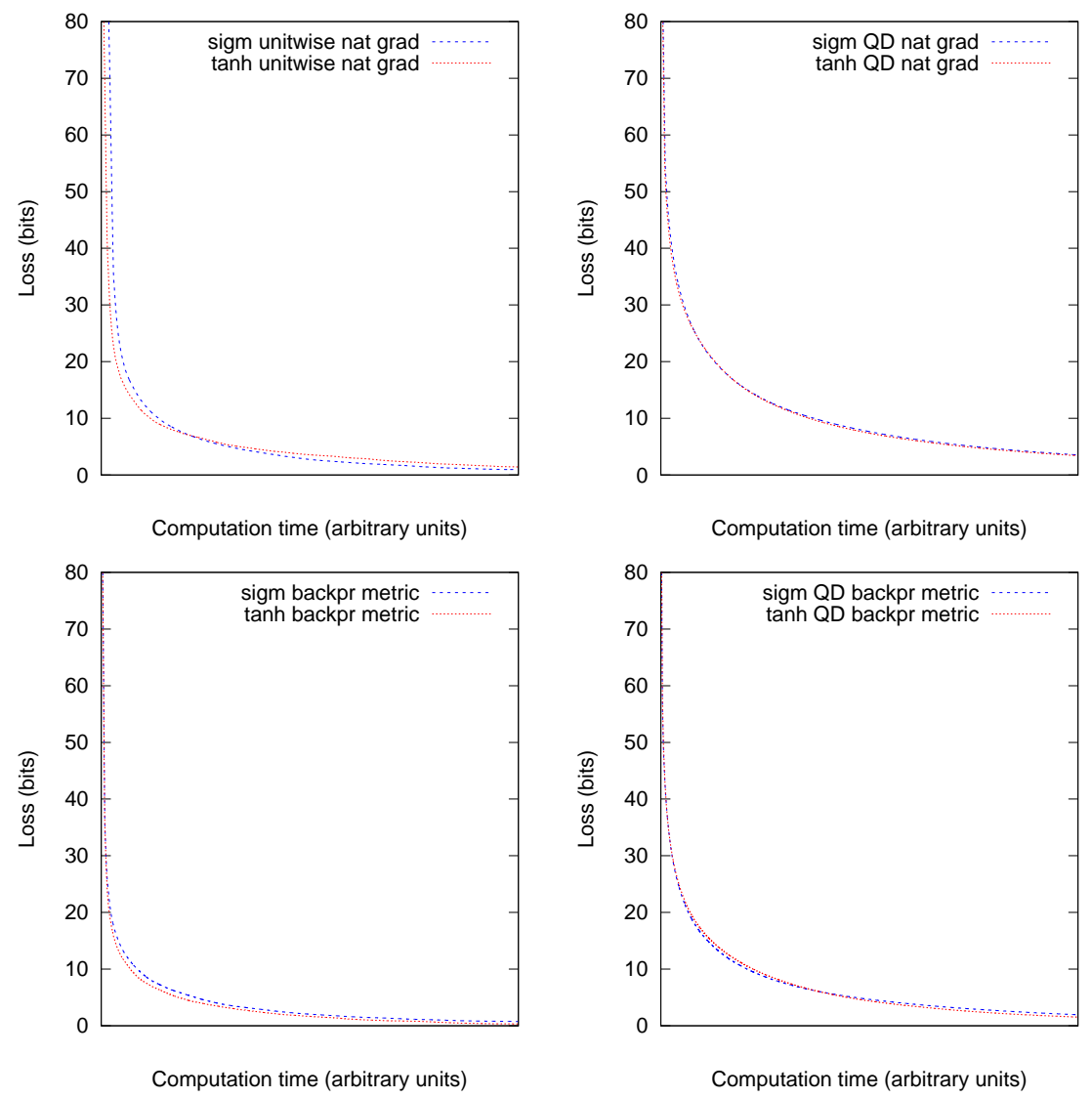

Figure 4: Double-checking invariance: Comparison of the trajectories of the invariant algorithms in tanh and sigmoid implementations 


\begin{tabular}{lcc}
\multicolumn{1}{c}{ Method } & \multicolumn{2}{c}{ Average loss (bits) \pm std-dev } \\
\cline { 2 - 3 } & sigmoid & tanh \\
\hline Non-invariant: & $35.9 \pm 2.1$ & $24.7 \pm 2.2$ \\
Backpropagation & $11.6 \pm 2.5$ & $3.5 \pm 2.0$ \\
Diagonal Gauss-Newton & $51.1 \pm 3.3$ & $25.3 \pm 2.0$ \\
AdaGrad & & \\
Invariant: & $0.9 \pm 1$ & $1.4 \pm 1.8$ \\
Unitwise natural gradient & $3.5 \pm 1.2$ & $3.4 \pm 1.6$ \\
Quasi-diagonal natural gradient & $0.8 \pm 0.8$ & $0.3 \pm 0.5$ \\
Backpropagated metric & $1.9 \pm 1.2$ & $1.5 \pm 1.3$ \\
Quasi-diagonal backpropagated metric & $12.9 \pm 1.5$ & $14.1 \pm 2.2$ \\
Monte Carlo unitwise natural gradient & $7.9 \pm 2.3$ & $10.1 \pm 2.5$ \\
Monte Carlo quasi-diagonal natural gradient & $24.7 \pm 2.5$ & $28.5 \pm 3.4$ \\
Unitwise OP gradient &
\end{tabular}

Table 2: Average loss per sample (bits) after an execution time equivalent to 10,000 backpropagation passes, computed over 20 independent runs, together with standard deviation over the runs

putation time budget. Per iteration instead of computation time, the natural gradient methods perform better than the backpropagated metric methods, and we expect them to be more competitive for smaller output sizes, e.g., in classification tasks.

The two algorithms based on squared gradients, Adagrad and the unitwise OP gradient, both perform rather poorly in this setting. Adagrad differs from the unitwise OP gradient by using a diagonal approximation and introducing square roots, which breaks invariance, while the unitwise OP gradient is invariant and is meant to approximate the natural gradient. This is a surprise, as, for instance, methods close to the OP gradient have been found to perform well, e.g., in RMB07, or in Oll13 for recurrent networks. The small size of the dataset in our setting is not enough to explain this problem, as it does not seem to affect the other methods. This may be related to the large dimensionality of the output layer compared to the number of samples in our experiment (in contrast to [RMB07] or [1l13]), which damages the one-sample OP metric approximation of the natural gradient and could result in low-rank OP matrices. Indeed, reasoning on the full (whole-network) metric, the OP gradient contributes a matrix of rank 1 for each data sample (see (101)); on the other hand, the exact Fisher matrix contributes a sum of $n_{\text {out }}$ matrices of rank 1 for each data sample as can be seen from (102)-(103). Thus from a theoretical viewpoint the quality of the one-sample OP approximation of the natural gradient is likely to depend on output dimensionality. 


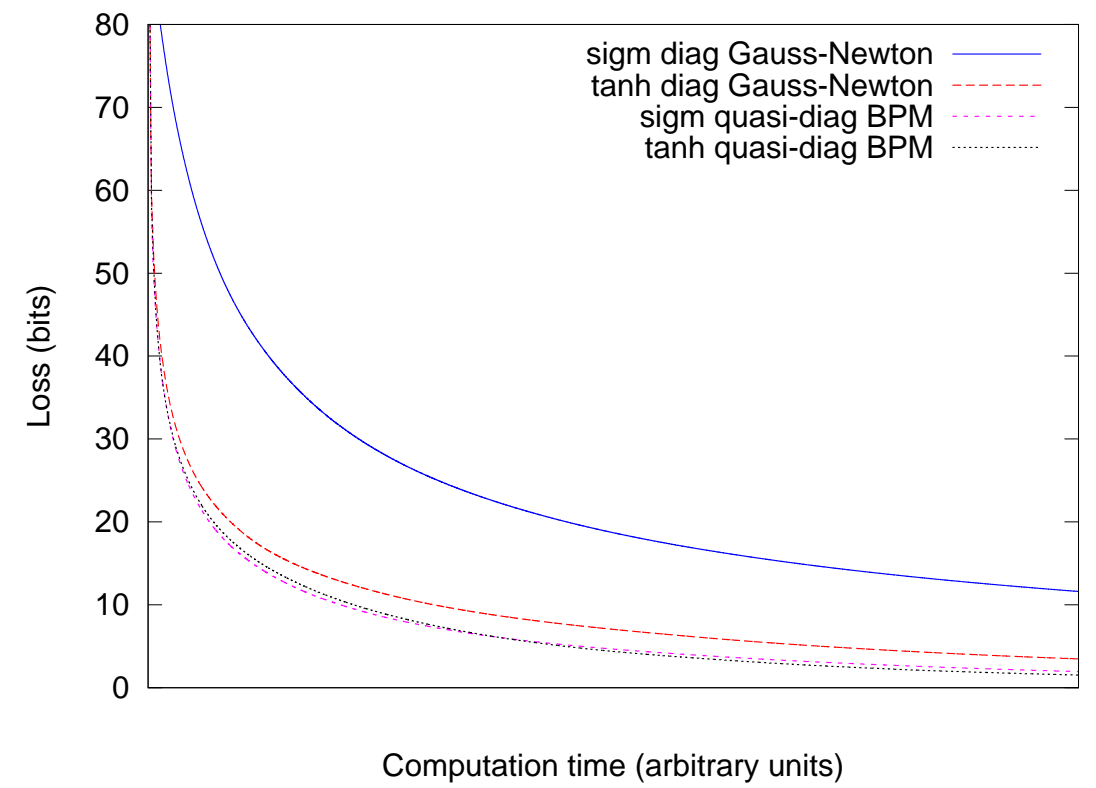

Figure 5: Effect of introducing a few non-diagonal terms: Comparison of the diagonal Gauss-Newton and the quasi-diagonal backpropagated metric methods

Lack of invariance is striking for some algorithms, such as the diagonal Gauss-Newton method: its performance is very different in the sigmoid and tanh interpretations (Figure 5). The quasi-diagonal backpropagated metric method only differs from diagonal Gauss-Newton by the inclusion of a small number of non-diagonal terms in the update. This change brings the sigmoid and tanh implementations in line with each other, improving performance with respect to the best of the two diagonal Gauss-Newton implementations. In settings where the activities of units (especially internal units, since the input can always be centered) are not as well centered as here, we expect the quasi-diagonal backpropagated metric method to outperform the tanh diagonal Gauss-Newton implementation even more clearly. Thus the quasidiagonal backpropagated metric is arguably "the invariant way" to write the diagonal Gauss-Newton algorithm.

In conclusion, although this experiment is a small-scale one, it clearly emphasizes the interest of using invariant algorithms.

\section{Conclusions}

- It is possible to write invariant training algorithms for neural networks that do not have the scalability issues of the natural gradient. For a 
network with $n$ units, $n_{\text {out }}$ output units, and at most $d$ incoming connections per unit, we have provided four invariant algorithms for which the cost of processing a data sample is respectively $O(n d), O\left(n d^{2}\right)$, $O\left(n d n_{\text {out }}\right)$ and $O\left(n d^{2}+n d n_{\text {out }}\right)$. The slower methods are closer to the natural gradient and have stronger invariance properties.

- All of these algorithms are mathematically motivated by building Riemannian metrics on the activity and parameter space of neural networks, treated as manifolds.

- The outer product metric encountered in the literature is also naturally interpreted in this framework. It has a unique property of spreading improvement most uniformly across the samples at each step.

- In a small-scale experiment involving an auto-encoding task with three hidden layers, invariant methods substantially outperform non-invariant methods. Lack of invariance is clear for some commonly used methods.

- The quasi-diagonal backpropagated metric is close to the diagonal Gauss-Newton method [LBOM96, SZL13] but crucially differs by the inclusion of a few well-chosen non-diagonal terms. While performance of the Gauss-Newton method can change substantially from sigmoid to tanh implementation, the quasi-diagonal backpropagated metric is invariant and improves performance with respect to diagonal GaussNewton in any implementation.

Acknowledgments. I would like to thank Youhei Akimoto, Jérémy Bensadon, Cécile Germain, Balázs Kégl, and Michèle Sebag for helpful conversations and their feedback on this article, as well as the anonymous referees for careful reading and helpful suggestions.

\section{Appendix}

\section{A Proof of Proposition 27}

The Fisher metric depends, of course, on the interpretation of the output layer as a probability distribution $\omega$. The final output $\omega$ is a probability distribution over the values of $y$ in the target space, parametrized by the activities $a_{k}$ of the output units $k$. If the output activities change by $\delta a_{k}$, the probability distribution $\omega$ will change as well. The norm of this change in Fisher metric is

$$
\begin{aligned}
\|\delta \omega\|_{\text {nat }}^{2} & =\mathbb{E}_{y \sim \omega}(\delta \ln \omega(y))^{2} \\
& =\sum_{k, k^{\prime} \in \mathcal{L}_{\text {out }}} \mathbb{E}_{y \sim \omega} \frac{\partial \ln \omega(y)}{\partial a_{k}} \frac{\partial \ln \omega(y)}{\partial a_{k^{\prime}}} \delta a_{k} \delta a_{k^{\prime}}
\end{aligned}
$$


thus stemming from the matrix

$$
F_{k k^{\prime}}:=\mathbb{E}_{y \sim \omega} \frac{\partial \ln \omega(y)}{\partial a_{k}} \frac{\partial \ln \omega(y)}{\partial a_{k^{\prime}}}
$$

over the output layer.

In the Bernoulli interpretation, for each component $k$ of the output layer, the random variable $y_{k}$ is a Bernoulli variable with parameter $a_{k}$. In the square-loss interpretation, each $y_{k}$ is a Gaussian random variable with mean $a_{k}$ and variance 1 . In the two classification interpretations, $y$ is a discrete random variable which takes value $k$ with probability $\omega_{k}=\mathrm{e}^{a_{k}} /\left(\sum_{j \in \mathcal{L}_{\text {out }}} \mathrm{e}^{a_{j}}\right)$ or $\omega_{k}=a_{k}^{2} /\left(\sum_{j \in \mathcal{L}_{\text {out }}} a_{j}^{2}\right)$.

Let us compute the Fisher metric in the space $\omega$ in each case. In the Bernoulli case, we have $\omega(y)=\prod_{k \in \mathcal{L}_{\text {out }}}\left(\mathbb{1}_{y_{k}=1} a_{k}+\mathbb{1}_{y_{k}=0}\left(1-a_{k}\right)\right)$. Consequently

$$
\frac{\partial \ln \omega(y)}{\partial a_{k}}=\frac{\mathbb{1}_{y_{k}=1}}{a_{k}}-\frac{\mathbb{1}_{y_{k}=0}}{1-a_{k}}=\frac{y_{i}-a_{k}}{a_{k}\left(1-a_{k}\right)}
$$

Since under the distribution $\omega$ we have $\mathbb{E} y_{k}=a_{k}$ and $\operatorname{Var} y_{k}=a_{k}\left(1-a_{k}\right)$ (with $y_{k}$ and $y_{j}$ independent for $k \neq j$ ) we find

$$
F_{k k^{\prime}}=\frac{\mathbb{1}_{k=k^{\prime}}}{a_{k}\left(1-a_{k}\right)}
$$

for $k$ and $k^{\prime}$ in the output layer.

In the Gaussian case we have $\omega(y)=\prod_{k} \frac{\mathrm{e}^{-\left(y_{i}-\omega_{k}\right)^{2} / 2}}{\sqrt{2 \pi}}$ so that $\frac{\partial \ln \omega(y)}{\partial \omega_{k}}=$ $y_{i}-\omega_{k}$. Since under the distribution $\omega$ we have $\mathbb{E} y_{k}=\omega_{k}$ and $\operatorname{Var} y_{k}=1$ we find $F=$ Id hence

$$
F_{k k^{\prime}}=\mathbb{1}_{k=k^{\prime}}
$$

for $k$ and $k^{\prime}$ in the output layer.

In the softmax case the probability to have $y=j$ is $\omega(y)=\mathrm{e}^{a_{j}} / S$ with $S=\sum_{i \in \mathcal{L}_{\text {out }}} \mathrm{e}^{a_{i}}$. Thus $\partial \ln \omega(y) / \partial a_{k}=\mathbb{1}_{k=j}-\mathrm{e}^{a_{k}} / S$. Taking the expectation over $y$ (i.e., over $j$ ) we find

$$
\begin{aligned}
F_{k k^{\prime}} & =\sum_{j \in \mathcal{L}_{\text {out }}} \frac{\mathrm{e}^{a_{j}}}{S}\left(\mathbb{1}_{j=k}-\frac{\mathrm{e}^{a_{k}}}{S}\right)\left(\mathbb{1}_{j=k^{\prime}}-\frac{\mathrm{e}^{a_{k^{\prime}}}}{S}\right) \\
& =\frac{\mathrm{e}^{a_{k}}}{S} \mathbb{1}_{k=k^{\prime}}-\frac{\mathrm{e}^{a_{k}}}{S} \frac{\mathrm{e}^{a_{k^{\prime}}}}{S}-\frac{\mathrm{e}^{a_{k^{\prime}}}}{S} \frac{\mathrm{e}^{a_{k}}}{S}+\left(\sum_{j} \frac{\mathrm{e}^{a_{j}}}{S}\right) \frac{\mathrm{e}^{a_{k}}}{S} \frac{\mathrm{e}^{a_{k^{\prime}}}}{S} \\
& =\frac{\mathrm{e}^{a_{k}}}{S} \mathbb{1}_{k=k^{\prime}}-\frac{\mathrm{e}^{a_{k}} \mathrm{e}^{a_{k^{\prime}}}}{S^{2}}
\end{aligned}
$$

Similarly, in the spherical case the probability to have $y=j$ is $\omega(y)=$ $a_{j}^{2} / S$ with $S=\sum_{i \in \mathcal{L}_{\text {out }}} a_{i}^{2}$. Thus $\partial \ln \omega(y) / \partial a_{k}=2\left(\frac{\mathbb{1}_{k=j}}{a_{j}}-\frac{a_{k}}{S}\right)$. Taking the 
expectation over $y$ we find

$$
\begin{aligned}
F_{k k^{\prime}} & =4 \sum_{j \in \mathcal{L}_{\text {out }}} \frac{a_{j}^{2}}{S}\left(\frac{\mathbb{1}_{j=k}}{a_{j}}-\frac{a_{k}}{S}\right)\left(\frac{\mathbb{1}_{j=k^{\prime}}}{a_{j}}-\frac{a_{k^{\prime}}}{S}\right) \\
& =4 \frac{a_{k}^{2}}{S} \frac{1}{a_{k}} \frac{1}{a_{k^{\prime}}} \mathbb{1}_{k=k^{\prime}}-4 \frac{a_{k}^{2}}{S} \frac{1}{a_{k}} \frac{a_{k^{\prime}}}{S}-4 \frac{a_{k^{\prime}}^{2}}{S} \frac{1}{a_{k^{\prime}}} \frac{a_{k}}{S}+4\left(\sum_{j} \frac{a_{j}^{2}}{S}\right) \frac{a_{k}}{S} \frac{a_{k^{\prime}}}{S} \\
& =\frac{4}{S} \mathbb{1}_{k=k^{\prime}}-\frac{4 a_{k} a_{k^{\prime}}}{S^{2}}
\end{aligned}
$$

These give the expression of the Fisher matrix $F_{k k^{\prime}}$ for $k$ and $k^{\prime}$ in the output layer. This is enough to compute the full Fisher matrix, as follows.

Let $x$ be an input for the network. Given a variation $\delta \theta$ of the network parameters $\theta$, let $\delta a_{i}$ be the resulting variation of unit $i$, and let $\delta \omega$ be the resulting variation of the final output $\omega$. We have $\delta \omega=\sum_{k \in \mathcal{L}_{\text {out }}} \frac{\partial \omega}{\partial a_{k}} \delta a_{k}$. The datum-wise Fisher metric on $\theta$ is

$$
\begin{aligned}
\|\delta \theta\|_{\text {nat }}^{2} & =\|\delta \omega\|_{\text {nat }}^{2} \\
& =\sum_{k, k^{\prime} \in \mathcal{L}_{\text {out }}} F_{k k^{\prime}} \delta a_{k} \delta a_{k^{\prime}}
\end{aligned}
$$

For each $k$ in the output layer, we have $\delta a_{k}=\sum_{i} \frac{\partial a_{k}}{\partial \theta_{i}} \delta \theta_{i}$ where the sum runs over all units $i$ in the network. For each $i$ we have $\frac{\partial a_{k}}{\partial \theta_{i}}=\frac{\partial a_{k}}{\partial a_{i}} \frac{\partial a_{i}}{\partial \theta_{i}}=J_{i}^{k} \frac{\partial a_{i}}{\partial \theta_{i}}$. Plugging this into the above yields

$$
\|\delta \theta\|_{\text {nat }}^{2}=\sum_{i} \sum_{i^{\prime}} \sum_{k \in \mathcal{L}_{\text {out }}} \sum_{k^{\prime} \in \mathcal{L}_{\text {out }}} F_{k k^{\prime}} J_{i}^{k} J_{i^{\prime}}^{k^{\prime}} \frac{\partial a_{i}}{\partial \theta_{i}} \frac{\partial a_{i^{\prime}}}{\partial \theta_{i^{\prime}}}
$$

so that the term of the Fisher matrix corresponding to $\delta \theta_{i}$ and $\delta \theta_{i^{\prime}}$ is $\sum_{k \in \mathcal{L}_{\text {out }}} \sum_{k^{\prime} \in \mathcal{L}_{\text {out }}} F_{k k^{\prime}} J_{i}^{k} J_{i^{\prime}}^{k^{\prime}} \frac{\partial a_{i}}{\partial \theta_{i}} \frac{\partial a_{i^{\prime}}}{\partial \theta_{i^{\prime}}}$.

For standard neural networks we have $\delta \theta_{i}=\left(\delta w_{j i}\right)_{j, j \rightarrow i}$ and moreover $\frac{\partial a_{i}}{\partial w_{j i}}=a_{j} r_{i}$.

Plugging into this the expression for $F_{k k^{\prime}}$ yields the results in Proposition 27.

\section{B Proof of Proposition 15}

Let $v$ be an infinitesimal variation of the parameter $\theta$. Let $v_{i}$ be the coordinates of $v$ in some coordinate system. At first order, the increment in the average loss function along $v$ is $\mathbb{E}_{x \in \mathcal{D}} \sum_{i} \frac{\partial \ell_{x}}{\partial \theta_{i}} v_{i}$.

Let us abbreviate $\partial_{i} \ell_{x}=\frac{\partial \ell_{x}}{\partial \theta_{i}}$. The matrix defining the OP metric is $M_{i j}=\mathbb{E}_{x \in \mathcal{D}} \partial_{i} \ell_{x} \partial_{j} \ell_{x}$. The corresponding gradient direction is $M^{-1} \mathbb{E}_{x \in \mathcal{D}} \partial \ell_{x}$.

Let $m=\mathbb{E}_{x \in \mathcal{D}} \sum_{i} \partial_{i} \ell_{x} v_{i}$ be the change in loss function associated with $v$. The variance, when $x$ runs over the dataset, of the gain in the loss function 
for $x$ is $\mathbb{E}_{x \in \mathcal{D}}\left(\left(\sum_{i} \partial_{i} \ell_{x} v_{i}\right)-m\right)^{2}=\mathbb{E}_{x \in \mathcal{D}}\left(\sum_{i} \partial_{i} \ell_{x} v_{i}\right)^{2}-m^{2}$. For fixed average gain $m$, this is minimal when $\mathbb{E}_{x \in \mathcal{D}}\left(\sum_{i} \partial_{i} \ell_{x} v_{i}\right)^{2}$ is minimal.

This is a smooth convex function of $v$, whose minimum we have to find over the hyperplane $\left\{v, \mathbb{E}_{x \in \mathcal{D}} \sum_{i} \partial_{i} \ell_{x} v_{i}=m\right\}$. The minimum of a positivedefinite quadratic functional $\sum_{i j} A_{i j} v_{i} v_{j}$ over a hyperplane $\sum_{i} B_{i} v_{i}=m$, is found at $v=\lambda A^{-1} B$ for some constant $\lambda$. Here we have $B_{i}=\mathbb{E}_{x \in \mathcal{D}} \partial_{i} \ell_{x}$, and expanding $\mathbb{E}_{x \in \mathcal{D}}\left(\sum_{i} \partial_{i} \ell_{x} v_{i}\right)^{2}=\mathbb{E}_{x \in \mathcal{D}}\left(\left(\sum_{i} \partial_{i} \ell_{x} v_{i}\right)\left(\sum_{j} \partial_{j} \ell_{x} v_{j}\right)\right)=\sum_{i j} \mathbb{E}_{x \in \mathcal{D}} \partial_{i} \ell_{x} \partial_{j} \ell_{x} v_{i} v_{j}$ yields $A_{i j}=\mathbb{E}_{x \in \mathcal{D}} \partial_{i} \ell_{x} \partial_{j} \ell_{x}=M_{i j}$. Consequently, for any value of $m$, the optimal $v$ is a multiple of the OP gradient direction $M^{-1} \mathbb{E}_{x \in \mathcal{D}} \partial \ell_{x}$.

\section{Definition of the metrics in the formalism of differential geometry}

Let $\mathcal{L}$ be the neural network (directed acyclic finite graph of units); for $k \in \mathcal{L}$, let the activities of unit $k$ belong to a manifold $\mathcal{A}_{k}$. Let the activation function for unit $k$ be $f_{k}:\left(\Theta_{k} \times \prod_{i \rightarrow k} \mathcal{A}_{i}\right) \rightarrow \mathcal{A}_{k},\left(\theta_{k},\left(a_{i}\right)_{i \rightarrow k}\right) \mapsto$ $f_{\theta_{k}}\left(\left(a_{i}\right)_{i \rightarrow k}\right)$ where $\Theta_{k}$ is the manifold of the parameters of unit $k$. Let $\mathcal{L}_{\text {in }} \subset \mathcal{L}$ and $\mathcal{L}_{\text {out }} \subset \mathcal{L}$ be the input and output layers, respectively; let $\mathcal{X}$ be the space to which the inputs belong, and let $\iota: \mathcal{X} \rightarrow \prod_{k \in \mathcal{L}_{\text {in }}} \mathcal{A}_{k}$ be the input encoding. Let $\mathcal{O}$ be the manifold to which the outputs belong, and let $\omega: \prod_{k \in \mathcal{L}_{\text {out }}} \mathcal{A}_{k} \rightarrow \mathcal{O}$ be the output interpretation.

The values of $a_{k}$ and of the output $\omega$ can be seen as functions of the parameter $\theta=\left(\theta_{k}\right) \in \prod_{k} \Theta_{k}$ and the input $x$, by using the induction relations defined by the network: $a_{k}(\theta, x):=\iota(x)_{k}$ for $k \in \mathcal{L}_{\text {in }}, a_{k}(\theta, x):=$ $f_{k}\left(\theta_{k},\left(a_{i}(\theta, x)\right)_{i \rightarrow k}\right)$ for $k \notin \mathcal{L}_{\text {in }}$, and by abuse of notation, $\omega(\theta, x):=\omega\left(\left(a_{k}(\theta, x)\right)_{k \in \mathcal{L}_{\text {out }}}\right)$.

Let $\Omega$ be the output metric: a Riemannian metric on the output manifold $\mathcal{O}$, which to every vector $\delta \omega$ tangent to $\mathcal{O}$ at point $\omega \in \mathcal{O}$, associates its square norm $\Omega_{\omega}(\delta \omega, \delta \omega)$ in a bilinear way. An important example is the Fisher metric when $\mathcal{O}$ is a space of probability distributions.

Let $\Theta:=\prod_{k} \Theta_{k}$ be the parameter manifold. We are going to define the natural metric, unitwise natural metric, and backpropagated metric as Riemannian metrics on $\Theta$.

If $\varphi: E \rightarrow F$ is a linear map between vector spaces $E$ and $F$, and $g$ is a bilinear form on $F$, we define the bilinear form $g \circ \varphi$ on $E$ by

$$
g \circ \varphi:\left(e, e^{\prime}\right) \mapsto g\left(\varphi(e), \varphi\left(e^{\prime}\right)\right)
$$

for any two vectors $e, e^{\prime} \in E$. If $g$ is positive-semidefinite then so is $g \circ \varphi$.

We denote by $T_{p} M$ the tangent space to a manifold $M$ at a point $p \in M$. Recall [GHL87, 1.36] that if $h: M \rightarrow M^{\prime}$ is a smooth map between manifolds, its differential $\frac{\partial h}{\partial p}(p)$ at point $p \in M$ is a linear map from $T_{p} M$ to $T_{h(p)} M^{\prime}$.

Let $\theta \in \Theta$. For an input $x$, let $\frac{\partial \omega}{\partial \theta}(\theta, x)$ be the differential of the network output $\omega(\theta, x)$ with respect to $\theta$ : this is a linear map from $T_{\theta} \Theta$ to $T_{\omega(\theta, x)} \mathcal{O}$. 
Define the natural metric $g_{\text {nat }}$ as the bilinear form on $T_{\theta} \Theta$ for each $\theta$ given by

$$
g_{\mathrm{nat}}:=\frac{1}{\# \mathcal{D}} \sum_{x \in \mathcal{D}}\left(\Omega_{\omega(\theta, x)} \circ \frac{\partial \omega}{\partial \theta}(\theta, x)\right)
$$

where $x$ ranges over inputs in the dataset $\mathcal{D}$. By construction, this metric does not depend on any choice of parametrization and is thus intrinsic.

The unitwise natural metric is defined in a likewise manner except that it first breaks down the tangent vector $\delta \theta \in T_{\theta} \Theta$ into its components along each unit $k$ using that $\Theta=\prod_{k} \Theta_{k}$ and thus $T_{\theta} \Theta=\bigoplus_{k} T_{\theta_{k}} \Theta_{k}$. The effect is to make the components $\delta \theta_{k}$ orthogonal. Namely:

$$
g_{\mathrm{u}-\mathrm{nat}}(\delta \theta, \delta \theta):=\sum_{k} g_{\text {nat }}^{k}\left(\delta \theta_{k}, \delta \theta_{k}\right)
$$

where $\delta \theta=\bigoplus_{k} \delta \theta_{k}$, and where

$$
g_{\text {nat }}^{k}:=\frac{1}{\# \mathcal{D}} \sum_{x \in \mathcal{D}}\left(\Omega_{\omega(\theta, x)} \circ \frac{\partial \omega}{\partial \theta_{k}}(\theta, x)\right)
$$

is the natural metric on $\Theta_{k}$, with $\frac{\partial \omega}{\partial \theta_{k}}(\theta, x)$ the differential of the network output with respect to $\theta_{k}$, which is a linear map from $T_{\theta_{k}} \Theta_{k}$ to $T_{\omega(\theta, x)} \mathcal{O}$.

The backpropagated metric is defined by backward induction in the directed acyclic graph $\mathcal{L}$. First, for each input $x$ and each unit $k$, let us define a bilinear form $g_{\mathrm{bp}, x}^{\mathcal{A}_{k}}$ on the tangent space $T_{a_{k}(\theta, x)} \mathcal{A}_{k}$ to the activity at $k$. On the output layer let us set

$$
g_{\mathrm{bp}, x}^{\mathcal{A}_{k}}:=\Omega_{\omega(\theta, x)} \circ \frac{\partial \omega}{\partial a_{k}}\left(\left(a_{j}(\theta, x)\right)_{j \in \mathcal{L}_{\text {out }}}\right) \quad \text { for } k \in \mathcal{L}_{\text {out }}
$$

where $\frac{\partial \omega}{\partial a_{k}}\left(\left(a_{j}(\theta, x)\right)_{j \in \mathcal{L}_{\text {out }}}\right)$ is the differential of the output interpretation function $\omega: \prod_{j \in \mathcal{L}_{\text {out }}} \mathcal{A}_{j} \rightarrow \mathcal{O}$ with respect to $a_{k}$, which is a linear map from $T_{a_{k}(\theta, x)} \mathcal{A}_{k}$ to $T_{\omega(\theta, x)} \mathcal{O}$. Then this is backpropagated through the network: for each $k$ we define a bilinear form on $T_{a_{k}(\theta, x)} \mathcal{A}_{k}$ by

$$
g_{\mathrm{bp}, x}^{\mathcal{A}_{k}}:=\sum_{i, k \rightarrow i} g_{\mathrm{bp}, x}^{\mathcal{A}_{i}} \circ \frac{\partial f_{i}}{\partial a_{k}}\left(\theta_{i},\left(a_{j}(\theta, x)\right)_{j \rightarrow i}\right) \quad \text { for } k \notin \mathcal{L}_{\text {out }}
$$

with $f_{i}: \Theta_{i} \times \prod_{j \rightarrow i} \mathcal{A}_{j} \rightarrow \mathcal{A}_{i}$ the activation function of unit $i$. (If a unit is both an output unit and influences some other units, we add the two contributions.) This is transferred to a metric on $\Theta_{k}$ via

$$
g_{\mathrm{bp}, x}^{\Theta_{k}}:=g_{\mathrm{bp}, x}^{\mathcal{A}_{k}} \circ \frac{\partial f_{k}}{\partial \theta_{k}}\left(\theta_{k},\left(a_{j}(\theta, x)\right)_{j \rightarrow k}\right)
$$

Finally, letting again $\delta \theta=\bigoplus_{k} \delta \theta_{k}$ be a tangent vector to $\Theta$, define the backpropagated metric by

$$
g_{\mathrm{bp}}(\delta \theta, \delta \theta):=\frac{1}{\# \mathcal{D}} \sum_{x \in \mathcal{D}} \sum_{k} g_{\mathrm{bp}, x}^{\Theta_{k}}\left(\delta \theta_{k}, \delta \theta_{k}\right)
$$


which is a metric on $\Theta$.

Note that these metrics may be non-positive definite (e.g., if a parameter has no influence on the output).

Since these metrics have been defined using only intrinsic objects without choosing a parametrization of any of the manifolds $\Theta_{k}$, they are intrinsic (for a given output metric $\Omega$ ). Consequently, when working in explicit coordinates, the value of the norm of $\delta \theta$ is invariant with respect to any change of variables for each $\theta_{k}$ (diffeomorphism of $\Theta_{k}$ ). The natural metric has the additional property that it is invariant under changes of variables mixing the parameters of various units: its invariance group is $\operatorname{Diff}\left(\prod_{k} \Theta_{k}\right)$ whereas the invariance group is the smaller group $\prod_{k} \operatorname{Diff}\left(\Theta_{k}\right)$ for the unitwise natural metric and backpropagated metric.

\section{References}

[AGS05] Luigi Ambrosio, Nicola Gigli, and Giuseppe Savaré. Gradient flows in metric spaces and in the space of probability measures. Lectures in Mathematics ETH Zürich. Birkhäuser Verlag, Basel, 2005.

[Ama98] Shun-ichi Amari. Natural gradient works efficiently in learning. Neural Comput., 10:251-276, February 1998.

[AN00] Shun-ichi Amari and Hiroshi Nagaoka. Methods of information geometry, volume 191 of Translations of Mathematical Monographs. American Mathematical Society, Providence, RI, 2000. Translated from the 1993 Japanese original by Daishi Harada.

[APF00] Shun-ichi Amari, Hyeyoung Park, and Kenji Fukumizu. Adaptive method of realizing natural gradient learning for multilayer perceptrons. Neural Computation, 12(6):1399-1409, 2000.

[Bis06] Christopher M. Bishop. Pattern recognition and machine learning. Springer, 2006.

[BL88] Sue Becker and Yann LeCun. Improving the convergence of back-propagation learning with second order methods. Technical Report CRG-TR-88-5, Department of Computer Science, University of Toronto, 1988.

[DHS11] John C. Duchi, Elad Hazan, and Yoram Singer. Adaptive subgradient methods for online learning and stochastic optimization. Journal of Machine Learning Research, 12:2121-2159, 2011.

[GB10] Xavier Glorot and Yoshua Bengio. Understanding the difficulty of training deep feedforward neural networks. In International 
Conference on Artificial Intelligence and Statistics, pages 249$256,2010$.

[GHL87] S. Gallot, D. Hulin, and J. Lafontaine. Riemannian geometry. Universitext. Springer-Verlag, Berlin, 1987.

[Kur94] Takio Kurita. Iterative weighted least squares algorithms for neural networks classifiers. New Generation Comput., 12(4):375394, 1994.

[LBOM96] Yann LeCun, Léon Bottou, Genevieve B. Orr, and Klaus-Robert Müller. Efficient backprop. In Genevieve B. Orr and KlausRobert Müller, editors, Neural Networks: Tricks of the Trade, volume 1524 of Lecture Notes in Computer Science, pages 9-50. Springer, 1996.

$\left[\mathrm{LNC}^{+} 11\right]$ Quoc V. Le, Jiquan Ngiam, Adam Coates, Ahbik Lahiri, Bobby Prochnow, and Andrew Y. Ng. On optimization methods for deep learning. In Lise Getoor and Tobias Scheffer, editors, ICML, pages 265-272. Omnipress, 2011.

[OAAH11] Yann Ollivier, Ludovic Arnold, Anne Auger, and Nikolaus Hansen. Information-Geometric Optimization algorithms: A unifying picture via invariance principles. Preprint, arXiv:1106.3708v2, 2011.

[Oll13] Yann Ollivier. Riemannian metrics for neural networks II: recurrent networks and learning symbolic data sequences. Preprint, arXiv:1306.0514, 2013.

[PB13] Razvan Pascanu and Yoshua Bengio. Revisiting natural gradient for deep networks. Preprint, http://arxiv.org/abs/1301.3584, 2013.

[RMB07] Nicolas Le Roux, Pierre-Antoine Manzagol, and Yoshua Bengio. Topmoumoute online natural gradient algorithm. In John C. Platt, Daphne Koller, Yoram Singer, and Sam T. Roweis, editors, NIPS. Curran Associates, Inc., 2007.

[RN03] Stuart Russell and Peter Norvig. Artificial Intelligence: A Modern Approach. Prentice-Hall, Englewood Cliffs, NJ, 2nd edition, 2003.

[SZL13] Tom Schaul, Sixin Zhang, and Yann LeCun. No more pesky learning rates. In Proc. International Conference on Machine learning (ICML'13), 2013. 